Portland State University

PDXScholar

2-12-1993

\title{
Exact Modeling of Time-Interval-Modulated Switched Networks
}

Weihe Niu

Portland State University

Follow this and additional works at: https://pdxscholar.library.pdx.edu/open_access_etds

Part of the Electrical and Electronics Commons

Let us know how access to this document benefits you.

\section{Recommended Citation}

Niu, Weihe, "Exact Modeling of Time-Interval-Modulated Switched Networks" (1993). Dissertations and Theses. Paper 4622.

https://doi.org/10.15760/etd.6506

This Thesis is brought to you for free and open access. It has been accepted for inclusion in Dissertations and Theses by an authorized administrator of PDXScholar. Please contact us if we can make this document more accessible: pdxscholar@pdx.edu. 
AN ABSTRACT OF THE THESIS OF Weihe Niu for the Master of Science in Electrical Engineering presented February 12, 1993

Title: Exact Modeling of Time-Interval-Modulated Switched Networks

APPROVED BY THE MEMBERS OF THE THESIS COMMITTEE:

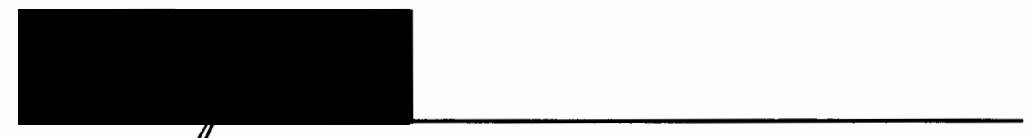
Richard Tymerski, Chair
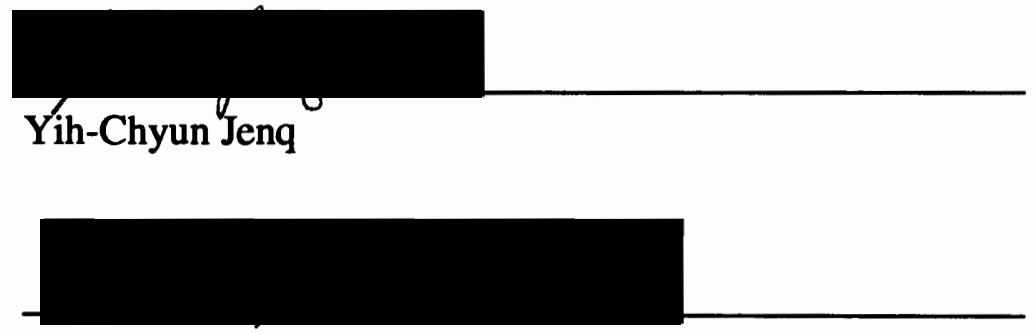

Dongik Joo

The frequency response analysis of switched networks plays a very important part in designing various kinds of power converter circuits. In this thesis two frequency response techniques for analyzing switching power converters are discussed. One method provides a mathematical description which treats the converter as a periodic time varying system. A linearized small signal model is subsequently derived. The major part of the thesis concentrates on this accurate exact small-signal technique. The derivation 
involves state space representation and the use of the time varying transfer function. A Fourier analysis is performed to show the relationship between the frequency response of the network and the time varying transfer function. The obtained expressions are in closed form. The method has proven to be exact. The complexity of this technique is overcome by automating its derivation in conjunction with a circuit simulator. An alternative method, relying only on a sampled-data representation, is also derived, which provides a less complicated algorithm. However the accuracy of this method suffers, particularly at high frequencies. The accuracy of the exact small-signal technique is verified by experimentation. 


\title{
EXACT MODELING OF TIME-INTERVAL-MODULATED SWITCHED NETWORKS
}

\author{
by \\ WEIHE NIU
}

\begin{abstract}
A thesis submitted in partial fulfillment of the requirements for the degree of
\end{abstract}

\author{
MASTER OF SCIENCE \\ in \\ ELECTRICAL ENGINEERING
}

Portland State University

1993 


\section{TO THE OFFICE OF GRADUATE STUDIES:}

The members of the Committee approve the thesis of Weihe Niu presented February 12, 1993.
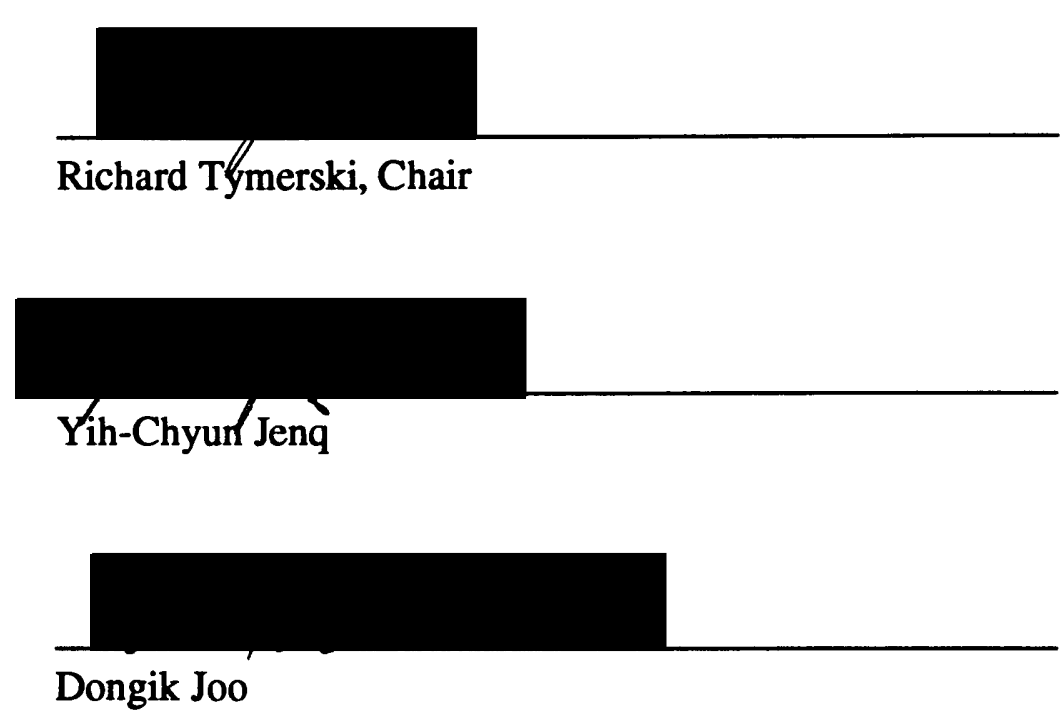

APPROVED:

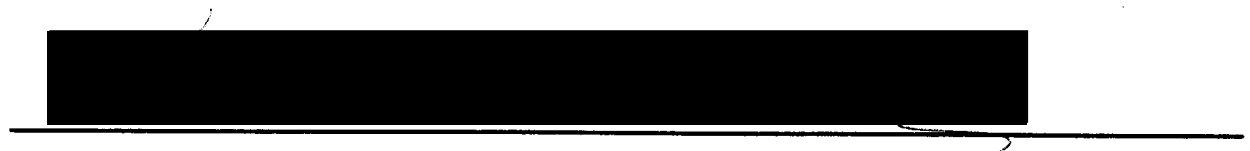

Rolf Schaumann, Chair, Department of Electrical Engineering

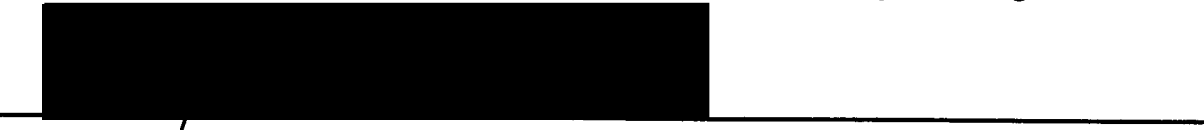

Roy W. Kqch, Vice Provost for Graduate Studies and Research 


\section{ACKNOWLEDGMENTS}

For the ever patient and helpful guidance through the various vicissitudes of this thesis, I would like to thank my advisor, Dr. Richard Tymerski, who enabled me, with his enormous experience in the fields of power electronics and control system design, to write the present thesis. Also it was only based on the theoretical and experimental work

he had previously performed that the thesis could be completed. Furthermore, I want to express my gratitude towards Lorraine Duncan, David Hansen, Shahin Pourkhesali and other staff members of the Computer Resources Laboratory of FRC, who always gave me great support in overcoming the difficulties I encountered when using the software to write the thesis. 
TABLE OF CONTENTS

PAGE

ACKNOWLEDGMENTS $\ldots \ldots \ldots \ldots \ldots \ldots \ldots \ldots \ldots \ldots \ldots \ldots \ldots$ iii

LIST OF FIGURES $\ldots \ldots \ldots \ldots \ldots \ldots \ldots \ldots \ldots \ldots \ldots \ldots \ldots \ldots, \mathbf{v}$

CHAPTER

I INTRODUCTION $\ldots \ldots \ldots \ldots \ldots \ldots \ldots \ldots \ldots \ldots \ldots \ldots, 1$

II MATHEMATICAL DESCRIPTION OF SWITCHED

NETWORKS ...........................

III SAMPLED-DATA REPRESENTATION ................. 19

IV THE EXACT SMALL-SIGNAL TECHNIQUE ........... 24

The Time Varying Transfer Function .............. 24

The Application of Time Varying Transfer Function ....... 30

The Derivation of Transfer Functions by Using

the Exact Small-Signal Technique........... 36

The Verification of the Results ............. 44

V CONCLUSION .......................... 57

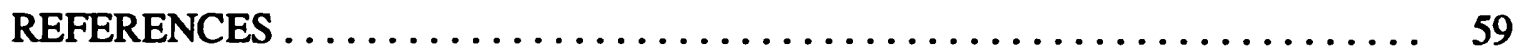




\section{LIST OF FIGURES}

1 A Feedback DC-to- DC Conversion System. . . . . . . . . .

2 Determining the Effect of the Output Current Variation $i_{o}$

on the Output Voltage $\hat{\nu}_{o}$ by Using a Perturbation

Source $\hat{i}_{g}\left(=\hat{i}_{o}\right) \ldots \ldots \ldots \ldots \ldots \ldots \ldots \ldots \ldots \ldots$

3 The System Block Diagram for $i$-th Subinterval ........... 9

4 Timing for One Operating Cycle ................ 11

5 State and Switching Times of the $i$-th Subinterval......... 31

6 Waveform Showing Relationship between the Control Signal and Switching Period.................. 33

7 Program Flow Diagram of Control to Output Transfer Function . 45

8 Program Flow Diagram of Input to Output Transfer Function ... 46

9 The Circuit of a PWM Boost Converter Operating in DCM . . . 49

10 The Control to Output Frequency Response of the PWM

Boost Converter Operating in DCM Verified by Measurement. ....................... 50

11 The Control to Output Frequency Response of the PWM

Boost Converter Operating in DCM ........... 51

12 The Input to Output Frequency Response of the PWM

Boost Converter Operating in DCM. .......... 52

13 The Circuit of a Current Programmed PWM Boost Converter

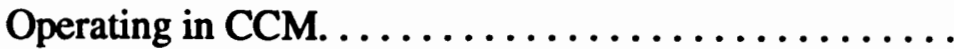


14 The Control to Output Frequency Response of the Current Programmed PWM Boost Converter Operating in CCM Verified by Measurement............... 54

15 The Control to Output Frequency Response of the Current Programmed PWM Boost Converter Operating in

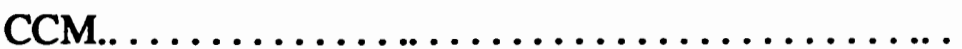

16 The Input to Output Frequency Response of the Current Programmed PWM Boost Converter Operating in CCM . . 


\section{CHAPTER I}

\section{INTRODUCTION}

Power processing by use of dc-to-dc switching converters is an important power conversion form. Circuit topologies used for dc-to-dc power converters form a basis from which other types of switching converter circuits such as ac-to-dc, dc-to-ac and acto-ac power converters may be derived. All forms of power processing circuitry are simply time-interval modulated switched networks. As high performance power processors, these converters are required to achieve a high efficiency, an acceptable transient response and an ability to overcome external disturbances. As with most systems where accuracy is required in the performance specifications, a dc-to-dc converter is usually enclosed in a feedback loop. Figure 1 shows a schematic representation of the closed loop dc-to-dc converter system. The output voltage $v_{o}$ is sensed and compared with a reference voltage $V_{\text {ref }}$. The difference signal $v_{\varepsilon}$ is amplified and the output signal of the amplifier $v_{c}$ is processed by the modulator. The modulator converts $v_{c}$ to a form which may be used to control the output voltage of the converter. The purpose of the feedback loop is to counteract any disturbance in the power source voltage $v_{s}$ and the load current $i_{\sigma}$. In order to derive the transfer functions of the network we need to decompose the instantaneous signals to find the small signal deviation terms, and then linearize these terms to build a small signal model so that a mathematical description can be found. The specifications of power converter systems usually dictate satisfying the following two criteria. Output voltage variations caused by variations of the input voltage must be below certain level over a specified frequency range. A transfer 


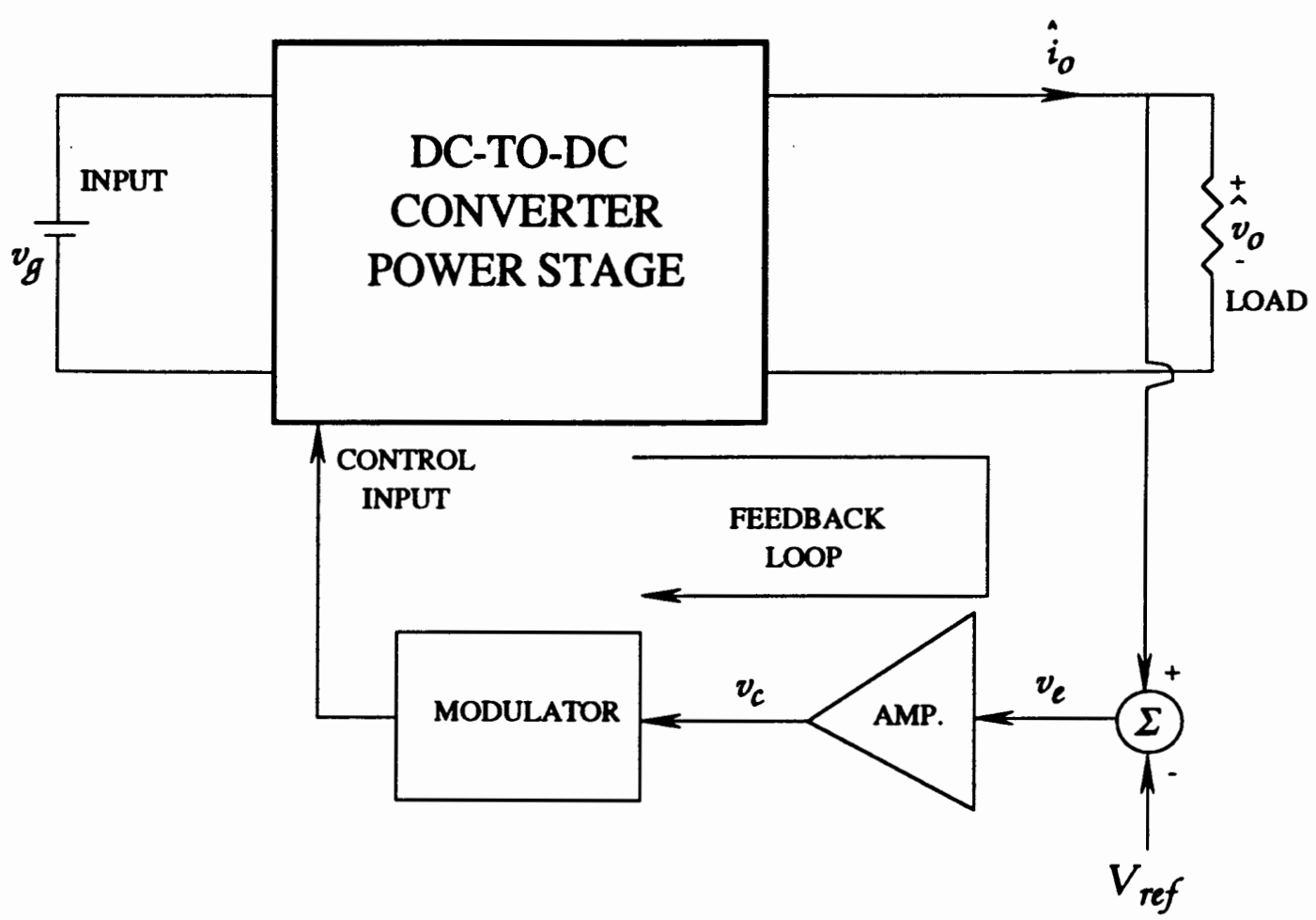

Figure 1. A feedback dc-to-dc conversion system. 
function $\hat{v}_{o}(s) / \hat{v}_{g}(s)$ is defined to investigate the relevant dynamic property. Another performance criterion is the requirement that the effect of load current variations on the output voltage is below a certain factor over a specific frequency range. We can simulate a perturbation current source $\hat{i}_{z}$ across the load resistor, as shown in Figure 2. The transfer function describing this property is defined as $\hat{v}_{o}(s) / \hat{i}_{g}(s)$. This criterion therefore relates to the magnitude of the output impedance being below a certain level over a specific frequency range. Both transfer functions depend heavily on the loop gain of the system. The loop gain includes an important transfer function, the relationship between control signal and output voltage $\hat{v}_{o}(s) / \hat{v}_{d}(s)$. From Figure 1 we can see that the error signal $v_{c}$ is amplified and used to control the converter power stage to counteract the effect of the external interference which has caused the output voltage variation. This thesis concentrates on the frequency response analysis of the open loop transfer functions of converter power stages. Both control to output and input to output open loop transfer functions will be investigated. The results provide Bode plots of the relevant responses. This graphic representation is easy to use in further analysis.

Presently there are a wide variety of techniques for the frequency analysis of power converters. However an accurate method over any arbitrary frequency range is yet to be developed, which will be the objective of Chapter IV of this thesis. Chapter IV extends the work of [ 1 ] and [ 2 ] results which can be applied to analyze any periodic time varying piecewise linear system.

A switched network is controlled by a switching device. It may involve several topologies. In power electronics the term "topology" is equivalent to "circuit". The time varying nature of a switched network is inherent. Any variation of switching times caused by external interference will affect state variables of the network. As we will see in later discussion, the exact small signal technique provides us with a frequency response with unparalleled accuracy. However the complexity of the method may be 


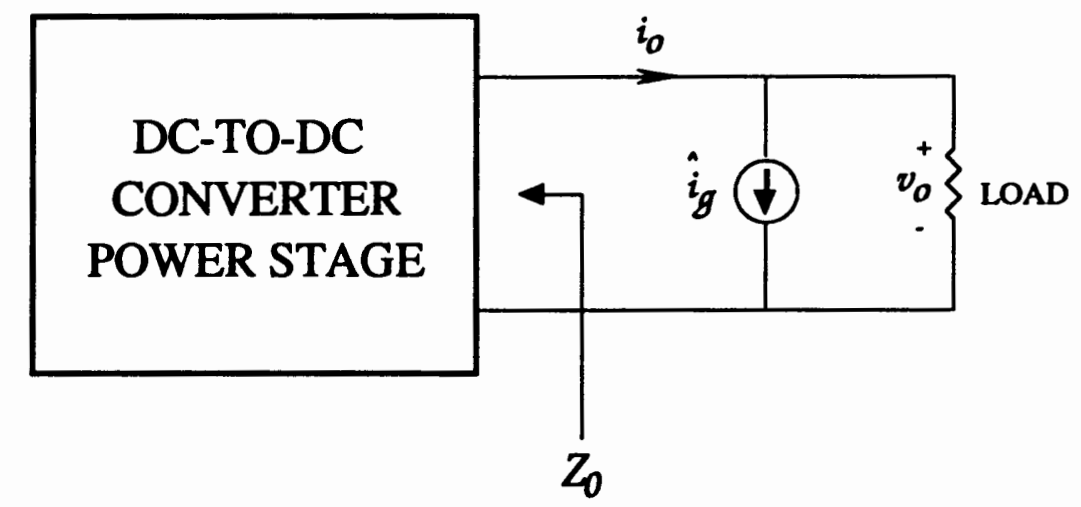

Eigure 2. Determining the effect of output current variations $\hat{i}_{o}$ on the output voltage $\hat{v}_{o}$ by using a perturbation source $\hat{i}_{g}\left(=\hat{i}_{o}\right)$. 
seen to be a drawback in certain instances. Some converter designers may be satisfied with a variety of relatively simpler methods. One of them is the state space averaging method, which presents either a mathematical description or an equivalent circuit model. The choice of which route to follow is a matter of practical convenience. The basic concept of this method is to avoid the "multi - topology" problem. In order to reduce ripples in output dc voltage, the converter generally has a filter in its output stage, which has a large time constant. The converter usually operates at a very high frequency, that means, its switching period is much shorter than the time constant of the output filter. Since the switching ripple can be neglected, it is possible, by applying a linear approximation, to find a single circuit model which contains comprehensive information of the dynamic property for all involved topologies. Since this technique represents only an approximation of the actual system it results in inaccuracy and its application is limited. Besides the exact method an alternative method which utilizes a discrete or sampled-data representation is presented in Chapter III. The switched network is regarded as being sampled at the switching frequency rate. The word "sampling" used in this case means that only the states at the switching instants are evaluated. The basic idea of the sampled-data representation is to find the difference equation relationship between two successive samples of the perturbed signal. The merit of this discrete technique is that the complexity of taking account of all details occurring between two successive samples is avoided, thus the derivation is significantly simplified. However, the sampling theorem applies in this case, which says that the sampling frequency should be at least twice the highest frequency in the signal to attain an non-aliasing signal reconstruction. If the sampling frequency is fixed, the highest frequency component of the signal which can be accurately reconstructed is half of the sampling frequency. The drawback of the sampled-data representation is that the upper limit of the frequency range is restricted. 
In the next chapter we will derive a small signal model, which will be applied in the derivations of both the sampled-data and the exact small-signal frequency analysis techniques. 


\section{CHAPTER II}

\section{MATHEMATICAL DESCRIPTION OF SWITCHED NETWORKS}

In this chapter we will find a mathematical description for general switched networks. The basic objective is to derive a linearized small signal model and an expression for calculating the steady state operating points. Looking at the equivalent circuit of a switching power converter we see that a series of different topologies emerge consecutively, and the whole process repeats itself. A topology changeover is not solely governed by the switching device. This particular switching operation is different from that of ordinary switching circuits, which are either in an ON or an OFF state. The number of topologies can be more than two. Another important property, the periodicity of the switched network, enable us to investigate the system in a limited time period. The information included in such a time period is sufficient for a complete dynamic description of the network. We can divide this period into several subintervals. Each topology is active within its corresponding subinterval. We define an integer value $N_{s}$ as the number of the topologies included in one time period. The following is a general state space representation for a typical time varying system

$$
\begin{aligned}
& \dot{x}(t)=A(t) x(t)+B(t) u(t) \\
& y(t)=C^{T}(t) x(t)+E(t) u(t)
\end{aligned}
$$

When the network is periodic and piecewise linear, it is possible to write a set of linear time invariant (LTI) state equations. Each one is valid in the corresponding subinterval. Thus the following expression is an alternate representation of ( $2-1)$ 


$$
\begin{aligned}
\dot{x}(t)= & A_{t} x(t)+B_{i} u(t) \\
y(t)= & C_{i}^{T} x(t)+E_{i} u(t) \\
& \text { for } t_{i}<t<t_{t+1}, \quad i \in\left(1,2, \ldots, N_{,}\right)
\end{aligned}
$$

The network description deals with a number of topologies. Figure 3 shows the block diagram of such a topology described by an LTI state space representation. Note that this representation of the network presents no information on how the control input signal $r(t)$ affects the states. The input matrix $B_{t}$ describes only the relationship between the states and the input $u(t)$, which is the fluctuating power source voltage, and $E_{t}$ relates $u(t)$ to the output. The control input signal $r(t)$ is basically a time quantity, which directly controls the time duration in which the switching device is "ON" or "OFF". By varying the time length during which a certain topology is active, this control signal may change the energy stored in some capacitor or inductor, and thus affect the states. Since the control signal has such a special form, we will find that when writing the differential equations it's difficult to find proper input and output matrices $B_{i}$ and $E_{i}$ to handle such a time quantity input signal. However we can consider an alternative to solve the problem. We can use the state equations ( $2-2 a)$ and $(2-2 b)$ and find its solution. As shown in Figure 3 the state equation has the form $\dot{x}=f(x, u)$. Since the solution of the equation, state $x(t)$ is a function of time $t$, and the control signal is also a time variation, it is possible to find the relationship between the states and the control input signal. The matrices $B_{i}$ and $E_{i}$ obtained don't deal with the control signal. The solution of the differential equation $x\left(t_{i}\right)$ is a function of time $t$. Since control signal is also a time quantity, the state $x\left(t_{i}\right)$ can be expressed as a time function.

The frequency response analysis is derived from a small signal model. We need to separate instantaneous signals into steady state terms and small perturbations. The control signal $r(t)$ can be expressed as 


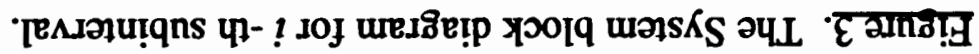

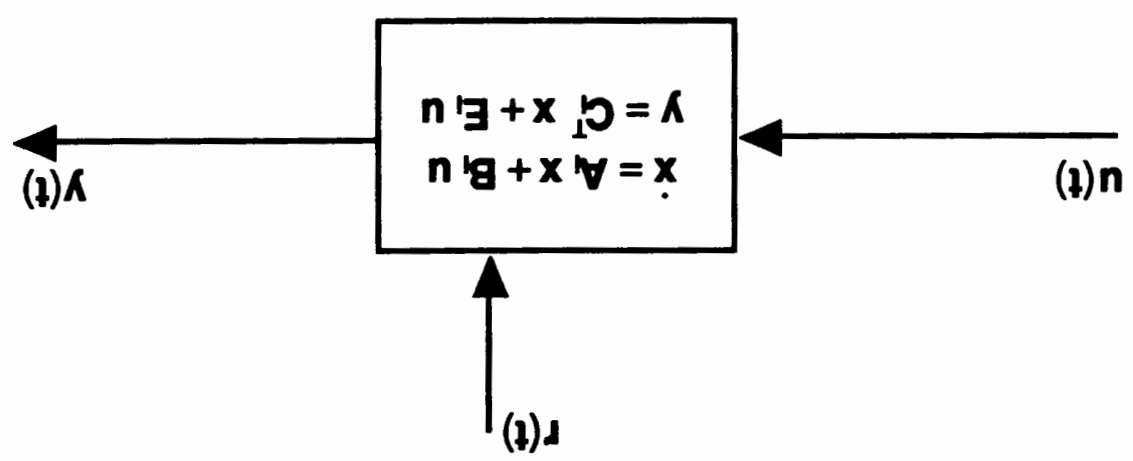




$$
r(t)=R+\hat{r}_{\mathrm{k}}(t)=R+\hat{r}_{p} e^{j \omega t}
$$

All those terms with a caret refer to the small perturbations, and the capitalized terms refer to steady state components. The perturbation term has a sinewave form. With such an assumption for the input signal it will be convenient to find the its relationship with output. The fluctuating input voltage is expressed in same way.

$$
u(t)=V_{s}+\hat{v}_{g}(t)=V_{s}+\hat{u e}^{j \omega t}
$$

There are other variables involved in later derivation. The time $t_{i}$, denotes the time instant at which a topology changeover occurs. It can be separated as

$$
t_{i}=T_{i}+\dot{t}_{i}
$$

If there is no disturbance the topology changeover will occur at the time instant $T_{i}$. Otherwise it may occur earlier or later by a small time shift $\dot{t}_{i}$. Note that $\dot{t}_{i}$ can be less than zero. The following expressions for state variables also hold.

$$
\begin{aligned}
& x\left(T_{i}\right)=X\left(T_{i}\right)+\hat{x}\left(T_{i}\right) \\
& x\left(t_{i}\right)=X\left(t_{i}\right)+\hat{x}\left(t_{i}\right)
\end{aligned}
$$

Substituting ( 2 - 5 ) in ( 2 - $6 \mathrm{~b})$ gives

$$
x\left(t_{i}\right)=X\left(T_{i}+\dot{t}_{i}\right)+\hat{x}\left(T_{i}+\hat{t}_{i}\right)
$$

After performing Taylor expansion, for the first term at $X\left(T_{i}\right)$ and for the second term at $x\left(T_{i}\right)$ respectively, we find an approximation for $(2-7)$

$$
x\left(t_{i}\right)=X\left(T_{i}\right)+\dot{X}\left(T_{i}\right) \dot{t}_{i}+\hat{x}\left(T_{i}\right)+\dot{\hat{x}}\left(T_{i}\right) \dot{t}_{i}
$$

The first term is the steady state. The second and third terms are first order perturbations. These are the variables we will use to build the small signal model. The last term is a product of perturbations. For small signals we can ignore this high order small quantity. Then

$$
x\left(t_{i}\right)=X\left(T_{1}\right)+\dot{X}\left(T_{i}\right) \dot{t}_{1}+\hat{x}\left(T_{i}\right)
$$

The switched network is a piecewise linear system. We have assumed that the number of subintervals contained in one operating cycle is $N_{s}$. Figure 4 illustrates the 


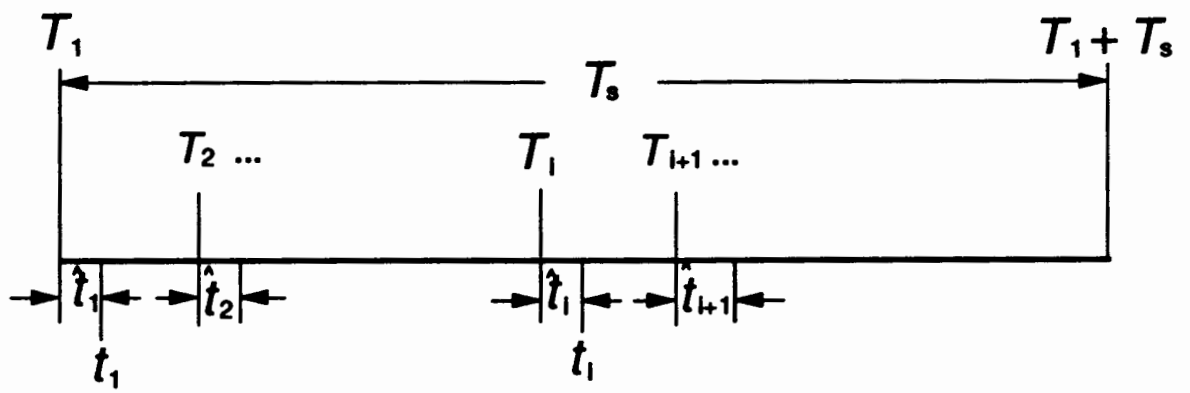

Eigure 4. Timing for one operating cycle. 
timing of the whole switching period $T_{s}$. As shown in Figure 4, each subinterval experiences a time perturbation $\hat{t}_{i}$, based on the steady state switching time $T_{i}$ $i \in\left(1,2, \ldots, N_{0}\right)$. This is a general case consideration. For a specific converter some of these time perturbations may not exist. In this case we can set them to zero. Now we can find the mathematical description for one subinterval. The state equation for the switched network, given by ( $2-2 a)$ and $(2-2 b)$, are rewritten

$$
\begin{aligned}
& \dot{x}(t)=A_{i} x(t)+B_{i} u(t) \\
& y(t)=C_{i}^{T} x(t)+E_{i} u(t) \\
& \text { for } t_{i}<t<t_{i+1} \quad i \in\left(1,2, \ldots, N_{s}\right)
\end{aligned}
$$

The solution of the state equation for the $i$ - th subinterval is given by

$$
x(t)=e^{A_{1}\left(t-t_{i}\right)} x\left(t_{i}\right)+\int_{t_{i}}^{t} e^{A_{i}(t-\tau)} B_{i} u(\tau) d \tau \quad \text { for } t_{i}<t<t_{i+1}
$$

The value of the state at the end of this subinterval can be calculated

$$
x\left(t_{i+1}\right)=e^{A\left(t_{i+1}-t_{i}\right)} x\left(t_{i}\right)+\int_{t_{1}}^{t_{t+1}} e^{A\left(t_{i+1}-\tau\right)} B_{i} u(\tau) d \tau
$$

The variables in expression ( $2-12$ ) are large signals. Since the time variables $t_{i}$ and $t_{i+1}$ are exponents, expression ( 2 - 12 ) is a non - linear expression. What we need is a linear, small signal model which gives the relationship between $\hat{x}\left(T_{i+1}\right)$ and $\hat{x}\left(T_{i}\right)$. Note that $T_{i+1}$ and $T_{i}$ may not be $t_{i+1}$ and $t_{i}$, the exact time instants the topology changeovers occur. Now we put the input signal, which is expressed in the form given by (2-4)

$$
u(t)=V_{e}+\hat{u} e^{j \omega t}
$$

in expression ( $2-12)$. Then

$$
x\left(t_{i+1}\right)=e^{A_{1}\left(t_{i+1}-t_{i}\right)} x\left(t_{i}\right)+\int_{t_{1}}^{t_{i+1}} e^{A_{1}\left(t_{i+1}-\tau\right)} B_{i} V_{s} d \tau+\int_{t_{i}}^{t_{i+1}} e^{A_{1}\left(t_{i+1}-\tau\right)} \hat{B}_{i} \hat{u} e^{j \omega \tau} d \tau
$$

Evaluating the integral yields

$$
x\left(t_{i+1}\right)=e^{A_{A}\left(t_{i+1}-t_{i}\right)} x\left(t_{i}\right)+\left(A_{i}^{-1}\right)\left(e^{\left.A_{(A+1}-t_{i}\right)}-I\right) B_{i} V_{z}
$$




$$
+\left(j \omega I-A_{i}\right)^{-1} e^{j \omega t_{t+1}}\left(I-e^{-\left(j \omega I-A_{i}\right)\left(t_{i+1}-t_{i}\right)}\right) B_{i} \hat{u}
$$

Then substituting $t_{i}=T_{1}+\hat{t}_{i}$ and $t_{t+1}=T_{i+1}+\hat{t}_{i+1}$ in the right side of $(2-14)$ gives

$$
\begin{aligned}
& x\left(T_{i+1}+\hat{t}_{i+1}\right)=e^{A_{i}\left[\left(T_{i+1}+\hat{t}_{i+1}\right)-\left(T_{i}+\hat{t}_{i}\right)\right]} x\left(T_{i}+\hat{t}_{i}\right) \\
& +\left(A_{i}\right)^{-1}\left(e^{A_{i}\left[\left(T_{i+1}+\hat{t}_{i+1}\right)-\left(T_{i}+\hat{t}_{i}\right)\right]}-I\right) B_{i} V_{8} \\
& +\left(j \omega I-A_{i}\right)^{-1} e^{j \omega\left(T_{i+1}+\hat{t}_{i+1}\right)}\left[I-e^{-(j \omega I-A)\left[\left(T_{t+1}+\hat{t}_{t+1}\right)-\left(T_{i}+\hat{t}_{i}\right)\right.}\right] B \hat{\mu} \\
& =e^{A_{i}\left[\left(T_{t+1}-T_{i}\right)-\left(\hat{t}_{t+1}-\hat{t}_{i}\right)\right]} x\left(T_{i}+\hat{t}_{i}\right)+\left(A_{i}\right)^{-1}\left(e^{A\left(T_{t+1}-T_{i}\right)} e^{A_{t}\left(\hat{i}_{t+1}-\hat{t}_{i}\right)}-I\right) B_{i} V_{s} \\
& +\left(j \omega I-A_{i}\right)^{-1} e^{j \omega T_{i+1}} e^{j \omega \hat{x}_{i+1}}\left[I-e^{-\left(j \omega I-A_{i}\right)\left(T_{i+1}-T_{i}\right)} e^{-\left(j \omega I-A_{i}\right)\left(\hat{t}_{t+1}-\hat{t}_{i}\right)}\right] B_{i} \hat{u}
\end{aligned}
$$

Expression $(2-15)$ is a nonlinear function of $\hat{t}_{i}$ and $\hat{t}_{t+1}$ because the time perturbations $\hat{t}_{i}$ and $\hat{t}_{t+1}$ appear as exponential terms. They can be linearized by replacing the exponential terms containing $\hat{t}_{i}$ and $\hat{t}_{t+1}$ with the zeroth and the first order terms of their Taylor expansion

$$
\hat{e^{\hat{x}}} \approx 1+\hat{x}
$$

Note that in $(2-9) \dot{X}\left(T_{i}\right)$ may be written as $A X\left(T_{i}\right)+B_{i} V_{8}$, such that

$$
x\left(T_{i}+\hat{t}_{i}\right)=X\left(T_{i}\right)+\dot{X}\left(T_{i}\right) \hat{t}_{i}+\hat{x}\left(T_{i}\right)=X\left(T_{i}\right)+\left[A_{i} X\left(T_{i}\right)+B_{i} V_{8}\right] \hat{t}_{i}+\hat{x}\left(T_{i}\right)
$$

and also

$$
x\left(T_{i+1}+\hat{t}_{i+1}\right)=X\left(T_{i+1}\right)+\left[A_{i+1} X\left(T_{i+1}\right)+B_{i+1} V_{z} \hat{t}_{i+1}+\hat{x}\left(T_{i+1}\right)\right.
$$

The expression $(2-15)$ can be written as

$$
\begin{aligned}
X\left(T_{i+1}\right) & +\left[A_{i+1} X\left(T_{i+1}\right)+B_{i+1} V_{z} \hat{t}_{i+1}+\hat{x}\left(T_{i+1}\right)\right. \\
& =e^{A\left(T_{i+1}-T_{i}\right)}\left[I+A_{i}\left(\hat{t}_{i+1}-\hat{t}_{i}\right)\right]\left[X\left(T_{i}\right)+\dot{X}\left(T_{i}\right) \hat{t_{i}}+\hat{x}\left(T_{i}\right)\right] \\
& +A_{i}^{-1}\left(e^{A_{f}\left(T_{t+1}-T_{i}\right)}\left[I+A_{i}\left(\hat{t}_{i+1}-\hat{t_{i}}\right)\right]-I\right) B_{i} V_{z} \\
& +\left[\left(j \omega I-A_{i}\right)^{-1} e^{j \omega T_{i+1}}\left(1+j \omega \hat{t}_{i+1}\right)\right]
\end{aligned}
$$


Now we drop the product of perturbation terms, which are second order quantities, and separate the zeroth order and first order terms in both sides of ( $2-19)$. Then a small signal model and an expression for steady states are obtained

$$
\begin{aligned}
\hat{x}\left(T_{i+1}\right)= & e^{A_{i}\left(T_{i+1}-T_{i}\right)} \hat{x}\left(T_{i}\right)+e^{A_{i}\left(T_{i+1}-T_{i}\right)}\left(A_{i} X\left(T_{i}\right)+B_{i} V_{g}\right) \hat{t}_{i+1} \\
& +\left(j \omega I-A_{i}\right){ }^{-1} e^{j \omega T_{i+1}}\left[I-e^{-\left(j \omega I-A_{i}\right)\left(T_{i+1}-T_{i}\right)}\right] \hat{B_{i} u} \\
& -A_{i+1} X\left(T_{i+1} \hat{x}_{i+1}-B_{i+1} V_{g} \hat{t}_{i+1}\right. \\
X\left(T_{i+1}\right) & =e^{A_{i}\left(T_{i+1}-T_{i}\right)} X\left(T_{i}\right)+A_{i}^{-1}\left(e^{A_{i}\left(T_{i+1}-T_{i}\right)}-I\right) B_{i} V_{g}
\end{aligned}
$$

In the second term of the right side of $(2-20)$, the coefficient of $\hat{t}_{i+1}$ can be written as

$$
\begin{aligned}
e^{A_{i}\left(T_{i+1}-T_{i}\right)} & \left(A_{i} X\left(T_{i}\right)+B_{i} V_{8}\right) \\
& =e^{A_{i}\left(T_{i+1}-T_{i}\right)} A_{i} X\left(T_{i}\right)+e^{A_{i}\left(T_{i+1}-T_{i}\right)} B_{i} V_{s}-B_{i} V_{s}+B_{i} V_{s} \\
& =e^{A_{i}\left(T_{i+1}-T_{i}\right)} A_{i} X\left(T_{i}\right)+\left(e^{A_{i}\left(T_{i+1}-T_{i}\right)}-I\right) B_{i} V_{s}+B_{i} V_{s} \\
& =A_{i}\left[e^{A_{i}\left(T_{i+1}-T_{i}\right)} X\left(T_{i}\right)+A_{i}^{-1}\left(e^{A_{i}\left(T_{i+1}-T_{i}\right)}-I\right) B_{i} V_{s}\right]+B_{i} V_{s} \\
& =A_{i} X\left(T_{i+1}\right)+B_{i} V_{8}
\end{aligned}
$$

Expression ( 2 - 20 ) becomes

$$
\begin{aligned}
\hat{x}\left(T_{i+1}\right) & =e^{A_{i}\left(T_{i+1}-T_{i}\right)} \hat{x}\left(T_{i}\right) \\
& +\left[\left(A_{i}-A_{i+1}\right) X\left(T_{i+1}\right)+\left(B_{i}-B_{i+1}\right) V_{s}\right] \hat{t}_{i+1} \\
& +\left(j \omega I-A_{i}\right)^{-1} e^{j \omega T_{i+1}}\left[I-e^{-\left(j \omega I-A_{i}\right)\left(T_{i+1}-T_{i}\right)}\right] \hat{B_{i} \hat{u}}
\end{aligned}
$$

Let $\Phi_{i}=e^{A_{1}\left(T_{i+1}-T_{i}\right)}$

$$
\begin{aligned}
& \xi_{i}=\left(A_{i}-A_{i+1}\right) X\left(T_{i+1}\right)+\left(B_{i}-B_{i+1}\right) V_{s} \\
& \eta_{i}=\left(j \omega I-A_{i}\right)^{-1}\left[I-e^{-\left(j \omega l-A_{i}\right)\left(T_{i+1}-T_{i}\right)}\right] B_{i} \\
& \hat{v}_{s}\left(T_{i+1}\right)=\hat{u} e^{j \omega T_{i+1}}
\end{aligned}
$$


Equation ( $2-23)$ can be expressed in a general form as

$$
\hat{x}\left(T_{i+1}\right)=\Phi_{i} \hat{x}\left(T_{i}\right)+\xi_{i} \hat{t}_{i+1}+\eta_{i} \hat{v}_{s}\left(T_{i+1}\right)
$$

A problem arises here that in finding the value of $\xi_{1}$ we need to know the value of the steady state $X\left(T_{i+1}\right)$. We have derived the steady state, which is given by ( $\left.2-21\right)$

$$
X\left(T_{i+1}\right)=e^{\left.A_{\uparrow} T_{i+1}-T_{i}\right)} X\left(T_{i}\right)+A_{i}^{-1}\left(e^{A_{f}\left(T_{i+1}-T_{i}\right)}-I\right) B_{i} V_{8}
$$

Expression ( 2 - 21 ) describes the behavior of the steady state $X\left(T_{i}\right)$. To find $X\left(T_{i+1}\right)$ we need to know the value of $X\left(T_{i}\right)$ first. For steady state values the following equation holds

$$
X\left(T_{i}\right)=X\left(T_{i}+T_{s}\right)
$$

Assume that $X\left(T_{1}\right)$ is the initial condition and we can find $X\left(T_{2}\right)$ by using $(2-21)$. Then using the expression of $X\left(T_{2}\right)$ as the initial condition we can find and express $X\left(T_{3}\right)$ in terms of $X\left(T_{1}\right)$ and input signals. Going through one whole operating cycle the expression of $X\left(T_{1}+T_{3}\right)$ can be obtained. Since $X\left(T_{1}\right)=X\left(T_{1}+T_{3}\right)$, by solving the equation obtained we can find the value of $X\left(T_{1}\right)$.

Expression ( $2-28$ ) is a difference equation, a mathematical description of the system in one subinterval. Note that this difference equation has a time quantity perturbation term $\xi_{i} i_{i+1}$. This time perturbation characterizes the time varying nature of the switched network. On one hand this time shift is partly caused by the control input signal, which affects the state. On the other hand any change of state and input signal may cause $\hat{t}_{t+1}$ to change. In fact $\hat{t}_{t+1}$ is an intermediate variable. It can be expressed as a linear combination of the state and input perturbations. Various kinds of switching converters differ significantly in their circuits. The relationships between $\hat{t}_{i+1}$ and other variables depend the circuit of specific converter. Before we solve the difference equation $\hat{t}_{i+1}$ must be evaluated first. From ( $\left.2-17\right)$ we can conclude that

$$
x\left(t_{i}\right)=X\left(T_{i}\right)+\left[A_{i} X\left(T_{i}\right)+B_{i} V_{g}\right] \hat{t}_{i}+\hat{x}\left(T_{i}\right)
$$

where equation ( 2 - 5 ) 


$$
t_{i}=T_{i}+\hat{t}_{i}
$$

is used in the left side of $(2-30)$. Note that

$$
x\left(t_{i}\right)=X\left(t_{i}\right)+\hat{x}\left(t_{i}\right)
$$

We pick only first order terms in ( 2 - 30 ) and find an equation for perturbation terms

$$
\hat{x}\left(t_{i}\right)=\left[A_{i} X\left(T_{i}\right)+B_{i} V_{s}\right] \hat{t_{i}}+\hat{x}\left(T_{i}\right)
$$

Changing the subscripts $t_{i}$ to $t_{i+1}$ results in

$$
\hat{x}\left(t_{i+1}\right)=\left[A_{i+1} X\left(T_{i+1}\right)+B_{i+1} V_{8}\right] \hat{t}_{i+1}+\hat{x}\left(T_{i+1}\right)
$$

By substituting ( 2 - 28 )

$$
\hat{x}\left(T_{i+1}\right)=\hat{\Phi} \hat{x}\left(T_{i}\right)+\hat{\xi}_{i} \hat{t}_{i+1}+\hat{\eta}_{i}\left(T_{i+1}\right)
$$

in ( 2 - 33 ) we can derive an expression for $\hat{x}\left(t_{i+1}\right)$

$$
\hat{x}\left(t_{i+1}\right)=\hat{\Phi_{i}} \hat{x}\left(T_{i}\right)+\left[A_{i} X\left(T_{i+1}\right)+B_{i} V_{s}\right] \hat{t}_{i+1}+\hat{\eta}_{i}\left(T_{i+1}\right)
$$

Let $\gamma_{i}=A_{i} X\left(T_{i+1}\right)+B_{i} V_{s}$. Then ( $\left.2-34\right)$ becomes

$$
\hat{x}\left(t_{i+1}\right)=\Phi_{i} \hat{x}\left(T_{i}\right)+\hat{\gamma}_{i} \hat{t}_{i+1}+\hat{\eta}_{i}\left(T_{i+1}\right)
$$

We will consider as a general controlled switching condition as follows, which is a current programming PWM converter operating in discontinuous conduction mode (DCM). The switching condition is satisfied when value of the control input $r(t)$, given in

$$
r\left(t_{i}\right)=R+\hat{r}_{k}\left(t_{i}\right)
$$

equals a linear combination of the states, $h_{i}^{T} x\left(t_{i+1}\right)$, plus a ramp with slope $m_{i}$. Thus

$$
r\left(t_{i+1}\right)=h_{i}^{T} x\left(t_{i+1}\right)+m_{i} t_{i+1}
$$

This form of switching condition accounts for a very wide variety of control techniques. Extracting the first order perturbation components of ( 2 - 36 ) we have

$$
\hat{r_{k}}\left(t_{i+1}\right)=\hat{h_{i}} \hat{x}\left(t_{i+1}\right)+m_{i} i_{i+1}
$$

Now we can express $\hat{t}_{i+1}$ in terms of $\hat{r}_{k}\left(t_{i+1}\right)$ and $\hat{x}\left(t_{i+1}\right)$. Substituting $(2-35)$ in 
( 2 - 37 ) and rearranging gives

$$
\hat{r_{k}}\left(t_{i+1}\right)=h_{i}^{T}\left[\hat{\Phi} \hat{x}\left(T_{i}\right)+\hat{\gamma}_{i} \hat{t}_{i+1}+\eta_{i} \hat{v}_{s}\left(T_{i+1}\right)\right]+m_{i} \hat{t}_{i+1}
$$

We consider this current programmed converter operating in DCM as our general case, which has $N_{s}$ topologies. We also assume that the control signal $\hat{r}(t)$, which appears only once during one switching cycle, is in effect at the instant $T_{N_{+}+1}$, the end of this cycle. The time perturbation $\hat{t}_{t+1}\left(i \in 1,2, \ldots N_{s}\right)$ can be evaluated as

$$
\begin{aligned}
& \hat{t}_{t+1}=-h_{i}{ }^{T} \mu_{i} \Phi_{i} \hat{x}\left(T_{i}\right)-h_{i}^{T} \mu_{i} \eta_{i} \hat{v}_{8}\left(T_{t+1}\right) \quad\left(i \in 1,2, \ldots, N_{s}-1\right) \\
& \hat{t}_{N_{f+1}}=\mu_{N_{s}} \hat{r}_{k}\left(T_{N_{s+1}}\right)-h_{N_{s}}^{T} \mu_{N_{s}} \Phi_{N_{s}} \hat{x}\left(T_{N_{s}}\right)-h_{N_{s}}^{T} \mu_{N_{s}} \eta_{N_{s}} \hat{v}_{s}\left(T_{N_{s+1}}\right)
\end{aligned}
$$

where $\mu_{i}=\frac{1}{h_{i}^{T} \gamma_{1}+m_{i}}$

The equation ( $2-39 \mathrm{~b}$ ), taking account of the existence of the control signal, is ture for the last subinterval. The expressions ( $2-39 \mathrm{a}$ ) give the values $\hat{t}_{t+1}$ for the rest $N_{\llcorner}-1$ subintervals. The values of parameters $\mu_{i}$ and $h_{1}^{\top}$ depend on the specific converter circuit and control method. Substituting $(2-39 a)$ and $(2-39 b)$ in $(2-28)$ results in

$$
\begin{aligned}
& \hat{x}\left(T_{i+1}\right)=\Phi_{i} \hat{x}\left(T_{i}\right)+\xi_{i}\left[-h_{i}^{T} \mu_{i} \hat{\Phi_{i}} \hat{x}\left(T_{i}\right)-h_{i}^{T} \mu_{i} \hat{\eta}_{i}\left(T_{i+1}\right)\right]+\hat{\eta_{i} \hat{v}_{s}}\left(T_{i+1}\right) \\
& =\Gamma_{i} \hat{\Phi_{i}} \widehat{x}\left(T_{i}\right)+\Gamma_{i} \hat{\eta}_{i}\left(T_{i+1}\right), \quad\left(i \in 1,2, \ldots, N_{s}-1\right) \\
& \hat{x}\left(T_{N_{s+1}}\right)=\Phi_{N_{s}} \hat{x}\left(T_{N_{s}}\right)+\xi_{N_{s}}\left(\mu_{N_{s}} \hat{r}_{k}\left(T_{N_{s+1}}\right)-h_{N_{s}}^{T} \mu_{N_{s}} \Phi_{N_{s}} \hat{x}\left(T_{N_{s}}\right)\right. \\
& \left.-h_{N_{s}}{ }^{T} \mu_{N_{s}} \eta_{N_{s}} \widehat{v_{s}}\left(T_{N_{s+1}}\right)\right)+\eta_{N_{s}} \hat{v}_{g}\left(T_{N_{s+1}}\right) \\
& =\Gamma_{N_{g}} \Phi_{N_{s}} \hat{x}\left(T_{N_{s}}\right)+\Gamma_{N_{s}} \eta_{N_{s}} \hat{v}_{g}\left(T_{N_{f+1}}\right)+\xi_{N_{s}} \mu_{N_{s}} \hat{r}_{k}\left(T_{N_{s+1}}\right)
\end{aligned}
$$

where $\Gamma_{i}=I-\xi_{i} h_{i}^{T} \mu_{i}$

We have obtained the desired relationship, for both small signal and the steady state quantities, between the final values and initial values for one subinterval. The following are a list of the important definitions and results given in this chapter.

$$
\Phi_{i}=e^{A_{i}\left(T_{H+1}-T_{i}\right)}
$$




$$
\begin{aligned}
& \xi_{i}=\left(A_{i}-A_{i+1}\right) X\left(T_{i+1}\right)+\left(B_{i}-B_{i+1}\right) V_{z} \\
& X\left(T_{i+1}\right)=e^{A_{f}\left(T_{i+1}-T_{i}\right)} X\left(T_{i}\right)+A_{i}^{-1}\left(e^{A_{f}\left(T_{i+1}-T_{i}\right)}-I\right) B_{i} V_{z} \\
& \eta_{t}=\left(j \omega I-A_{i}\right)^{-1}\left[I-e^{-\left(j \omega I-A_{i}\right)\left(T_{i+1}-T_{i}\right)}\right] B_{i} \\
& \hat{t}_{i+1}=-h_{i}^{T} \mu_{i} \Phi_{i} \hat{x}\left(T_{i}\right)-h_{i}^{T} \mu_{i} \eta_{i v_{s}}\left(T_{i+1}\right),(i \in 1,2, \ldots, N,-1) \\
& \hat{t}_{N_{s+1}}=\mu_{N_{s}} \hat{r}_{k}\left(T_{N_{+}+1}\right)-h_{N_{8}}^{T} \mu_{N_{s}} \Phi_{N_{s}} \hat{x}\left(T_{N_{s}}\right)-h_{N_{s}}^{T} \mu_{N_{s}} \eta_{N_{s}} \hat{v_{g}}\left(T_{N_{N_{+}+1}}\right) \\
& \mu_{i}=\frac{1}{h_{i}^{T} \gamma_{i}+m_{i}} \\
& \Gamma_{i}=I-\xi_{i} h_{i}{ }^{T} \mu_{i} \\
& \hat{x}\left(T_{i+1}\right)=\Gamma_{i} \hat{\Phi_{i}} \hat{x}\left(T_{i}\right)+\Gamma_{i} \hat{\eta}_{i v_{z}}\left(T_{i+1}\right),\left(i \in 1,2, \ldots, N_{,}-1\right) \\
& \hat{x}\left(T_{N_{s+1}}\right)=\Gamma_{N_{s}} \Phi_{N_{s}} \hat{x}\left(T_{N_{s}}\right)+\Gamma_{N_{s}} \eta_{N_{s}} \hat{v}_{8}\left(T_{N_{s+1}}\right)+\xi_{N_{s}} \mu_{N_{s}}, \hat{r}_{k}\left(T_{N_{N+1}}\right)
\end{aligned}
$$

For control and disturbance input, we define their perturbation terms having the following form

$$
\begin{aligned}
& \hat{r}_{k}(t)=\hat{r}_{p} e^{j \omega t} \\
& \hat{v}_{g}(t)=\hat{u} e^{j \omega t}
\end{aligned}
$$

The complexity of deriving and calculating the mathematical description for a time varying system is much greater than that of dealing with ordinary time-invariant system. A C++ program was written and applied to automate the calculation. A few examples using this program will be presented for the exact small-signal frequency analysis technique of Chapter IV. In this chapter the mathematical description can be programmed that once loaded with parameters of a specific switched network, the corresponding small signal model is readily presented for further analysis. 


\section{CHAPTER III}

\section{SAMPLED - DATA REPRESENTATION}

Sampled data representation describes the relationship between the state variable perturbations sampled at time instants $T_{I}$ and $T_{E}$. Here $T_{I}$ stands for the initial instant and $T_{E}$ for the end instant. The time interval $T_{E}-T_{I}$ is the steady state operating cycle $T_{3}$. For the convenience of the derivation we set $T_{l}$ at $k T$, a steady state switching instant, and set $T_{E}$ at $(k+1) T_{s}$. We also suppose that at the instant $k T_{s}$, the value of the state is $x(k T)$ and for $(k+1) T$, the state is $x[(k+1) T]$. The principle of sampled-data representation is finding the mathematical relationship between state perturbations $\hat{x}\left[(k+1) T_{0}\right]$ and $\hat{x}\left(k T_{1}\right)$. The relationship is a difference equation which results in a small signal model. Once the relation is obtained, by using the $\mathrm{Z}$ - transform we can solve the difference equation to find the solution $\hat{x}(z)$. Since $z=e^{j \omega T_{x}}, \hat{x}(z)$ can be expressed as a function of frequency. Note that only when there exist no external interferences the exact topology changeover may happen at their steady state time instants $k T$, and $(k+1) T$. . Otherwise the effect of the input signal may cause a time shift of the changeover instants. The actual operating interval may not be $T$.. To find the relationship between $\hat{x}[(k+1) T]$ and $\hat{x}(k T$, $)$, we can utilize the result previously obtained. In Chapter II we found the small signal mathematical description of the switched network in one subinterval. The state variables can be expressed by a linear combination of its initial value and external interferences, which are expressed by $(2-41 a)$ and $(2-41 b)$

$$
\begin{aligned}
& \hat{x}\left(T_{i+1}\right)=\Gamma_{i} \Phi_{i} \hat{x}\left(T_{i}\right)+\Gamma_{i} \eta_{i} \hat{v}_{s}\left(T_{t+1}\right), \quad\left(i \in 1,2, \ldots, N_{s}-1\right) \\
& \hat{x}\left(T_{N_{s+1}}\right)=\Gamma_{N_{s}} \Phi_{N_{s}} \hat{x}\left(T_{N_{s}}\right)+\Gamma_{N_{s}} \eta_{N_{s} \hat{v}_{s}}\left(T_{N_{s+1}}\right)+\xi_{N} \mu_{N_{s}} \hat{r}_{t}\left(T_{N_{s+1}}\right)
\end{aligned}
$$


We need to find this relationship for all $N_{s}$ subintervals, with the final value $\hat{x}\left[(k+1) T_{s}\right]$ obtained in current subinterval taken as the initial value $\hat{x}(k T$ s $)$ of the next subinterval. The following is an example for a 4 - subinterval case. We assume $\hat{t}_{i+1} \neq 0,(i \in 1,2,3,4)$. The conclusion derived in this case can be extended to a general case in which $N_{s}$ takes any integer value. Referred to expression ( 2 - 41a ) and ( 2 - $41 \mathrm{~b}$ ) the expressions for $N_{s}=4$ are

$$
\begin{aligned}
& \hat{x}\left(T_{i+1}\right)=\Gamma_{i} \hat{\Phi_{i} \hat{x}}\left(T_{i}\right)+\Gamma_{i} \eta_{i} \hat{v_{8}}\left(T_{i+1}\right), \quad(i \in 1,2,3) \\
& \hat{x}\left(T_{5}\right)=\Gamma_{4} \Phi_{4} \hat{x}\left(T_{4}\right)+\Gamma_{4} \eta_{4} \hat{v}_{8}\left(T_{5}\right)+\xi_{4} \mu \hat{r}_{k}\left(T_{3}\right)
\end{aligned}
$$

We suppose there is a state perturbation $\widehat{x}\left(T_{1}\right)$ at the beginning of the first subinterval, the instant $k T_{s}$, and the disturbance signals of all subintervals are $\hat{v}_{s}\left(T_{i+1}\right)(i \in 1,2,3,4)$. We start at the first subinterval to find $\widehat{x}\left(T_{2}\right)$

$$
\hat{x}\left(T_{2}\right)=\Gamma_{1} \hat{\Phi_{1} x\left(T_{1}\right)}+\Gamma_{1} \hat{\eta_{1} \hat{v}_{8}}\left(T_{2}\right)
$$

Now by taking $\widehat{x}\left(T_{2}\right)$ as the initial value of the second subinterval we can find $\widehat{x}\left(T_{3}\right)$, and further, $\hat{x}\left(T_{4}\right)$, all in terms of $\hat{x}\left(T_{1}\right)$ and involved input signals

$$
\begin{aligned}
& \widehat{x}\left(T_{3}\right)=\Gamma_{2} \hat{\Phi_{2} x}\left(T_{2}\right)+\Gamma_{2} \hat{\eta}_{2} \bar{v}_{8}\left(T_{3}\right) \\
& =\Gamma_{2} \Phi_{2} \Gamma_{1} \hat{\Phi_{2} \hat{x}}\left(T_{1}\right)+\Gamma_{2} \Phi_{2} \Gamma_{1} \hat{\eta_{1} v_{8}}\left(T_{2}\right)+\Gamma_{2} \hat{\eta_{2} v_{8}}\left(T_{3}\right) \\
& \hat{x}\left(T_{4}\right)=\Gamma_{3} \hat{\Phi_{3} \hat{x}}\left(T_{3}\right)+\Gamma_{3} \hat{\eta_{3} \nu_{8}}\left(T_{4}\right) \\
& =\Gamma_{3} \Phi_{3} \Gamma_{2} \Phi_{2} \Gamma_{1} \hat{\Phi_{1}} \hat{x}\left(T_{1}\right)+\Gamma_{3} \Phi_{3} \Gamma_{2} \Phi_{2} \Gamma_{1} \hat{\eta_{1} v_{8}}\left(T_{2}\right) \\
& +\Gamma_{3} \Phi_{3} \Gamma_{2} \hat{\eta_{2} \hat{v}_{8}}\left(T_{3}\right)+\Gamma_{3} \hat{\eta_{3}} \hat{v_{8}}\left(T_{4}\right)
\end{aligned}
$$

In the last subinterval the control signal $\hat{r_{k}}(t)$ is introduced

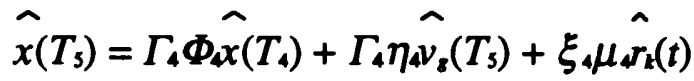

$$
\begin{aligned}
& =\Gamma_{4} \Phi_{4} \Gamma_{3} \Phi_{3} \Gamma_{2} \Phi_{2} \Gamma_{1} \Phi_{1} \hat{x}\left(T_{1}\right)+\Gamma_{4} \Phi_{4} \Gamma_{3} \Phi_{3} \Gamma_{2} \Phi_{2} \Gamma_{1} \eta_{1} v_{8}\left(T_{2}\right)
\end{aligned}
$$




$$
+\Gamma_{4} \Phi_{4} \Gamma_{3} \Phi_{3} \Gamma_{2} \hat{\eta}_{2} \hat{v}_{8}\left(T_{3}\right)+\Gamma_{4} \Phi_{4} \Gamma_{3} \hat{\eta}_{3} \hat{v}_{8}\left(T_{4}\right)+\Gamma_{4} \eta_{4} \hat{v}_{8}\left(T_{5}\right)+\xi_{4} \mu_{4} \hat{r}_{k}(t)
$$

Some parameters of the network and input signals have to be evaluated in order to make it easier to derive the difference equation. As defined earlier, the disturbance signals have a form

$$
\hat{v}_{8}\left(T_{i+1}\right)=\hat{u} e^{j \omega T_{i+1}}
$$

We define a parameter $\beta_{i}$

$$
\beta_{i}=e^{-j \omega\left(T_{i+1}-T_{i}\right)} \Phi_{i}=e^{-j \omega\left(T_{t+1}-T_{i}\right)} e^{A\left(T_{i+1}-T_{i}\right)}=e^{-\left(j \omega r-A_{i}\right)\left(T_{t+1}-T_{i}\right)}
$$

then the term of $\widehat{v_{s}}\left(T_{2}\right)$ can be expressed as

$$
\begin{aligned}
\Gamma_{4} \Phi_{4} \Gamma_{3} \Phi_{3} \Gamma_{2} \Phi_{2} \Gamma_{1} \eta_{1} \hat{\nu_{8}}\left(T_{2}\right)=\Gamma_{4} \Phi_{4} \Gamma_{3} \Phi_{3} \Gamma_{2} \Phi_{2} \Gamma_{1} \hat{\eta}_{u e^{j \omega T_{2}}} \\
=\Gamma_{4} e^{-j \omega\left(T_{5}-T_{4}\right)} \Phi_{4} \Gamma_{3} e^{-j \omega\left(T_{4}-T_{3}\right)} \Phi_{3} \Gamma_{2} e^{-j \omega\left(T_{3}-T_{2}\right)} \Phi_{2} \Gamma_{1} \eta_{1} \hat{u} e^{j \omega T_{5}} \\
=\Gamma_{4} \beta_{4} \Gamma_{3} \beta_{3} \Gamma_{2} \beta_{2} \Gamma_{1} \eta_{1} \hat{u} e^{j \omega T_{5}} \\
=\Gamma_{4} \beta_{4} \Gamma_{3} \beta_{3} \Gamma_{2} \beta_{2} \Gamma_{1} \eta_{1} \hat{v_{8}}\left(T_{5}\right)
\end{aligned}
$$

By using same technique we can do this for other terms

$$
\begin{aligned}
& \Gamma_{4} \Phi_{4} \Gamma_{3} \Phi_{3} \Gamma_{2} \eta_{2} \hat{v}_{8}\left(T_{3}\right)=\Gamma_{4} \beta_{4} \Gamma_{3} \beta_{3} \Gamma_{2} \eta_{2} \hat{v_{8}}\left(T_{5}\right) \\
& \Gamma_{4} \Phi_{4} \Gamma_{3} \eta_{3} \hat{v_{8}}\left(T_{4}\right)=\Gamma_{4} \beta_{4} \Gamma_{3} \eta_{3} \hat{v_{8}}\left(T_{5}\right)
\end{aligned}
$$

For the control signal, it has been assumed that $\hat{r}_{k}(t)$ has a form of $\hat{r}_{p} e^{j \omega t}$. We set $t=T_{s}$, the end of last subinterval, which is the instant $(k+1) T_{s}$. Then from the definition we can derive

$$
\begin{aligned}
& \left(\hat{r}_{k}(t)\right)_{t=T_{s}}=\hat{r}_{k}\left(T_{s}\right)=\hat{r}_{p} e^{j \omega T_{s}}=\hat{r}_{p} e^{j \omega(k+1) T_{s}}=\hat{r}_{p} e^{j \omega k T_{s}} e^{j \omega T_{s}}=\hat{r}_{k}\left(k T_{s}\right) e^{j \omega T_{s}} \\
& \hat{v}_{8}\left(T_{5}\right)=\hat{v}_{8} e^{j \omega k T} e^{j \omega T T_{s}}=\hat{v}_{8}\left(k T_{s}\right) e^{j \omega T_{s}}
\end{aligned}
$$

Since $T_{1}=k T_{s}, T_{s}=(k+1) T_{s}$, expression $(3-5)$ becomes

$$
\begin{aligned}
\hat{x}\left[(k+1) T_{3}\right] & =\Gamma_{4} \Phi_{4} \Gamma_{3} \Phi_{3} \Gamma_{2} \Phi_{2} \Gamma_{1} \hat{\Phi_{1}} \widehat{x}\left(k T_{3}\right) \\
& +\left[\left(\Gamma_{4} \beta_{4} \Gamma_{3} \beta_{3} \Gamma_{2} \beta_{2} \Gamma_{1} \eta_{1}+\Gamma_{4} \beta_{4} \Gamma_{3} \beta_{3} \Gamma_{2} \eta_{2}+\Gamma_{4} \beta_{4} \Gamma_{3} \eta_{3}+\Gamma_{4} \eta_{4}\right)\right.
\end{aligned}
$$




$$
\left.\hat{v_{s}}\left(k T_{s}\right)+\xi_{4} \hat{r}_{r_{k}}\left(k T_{s}\right)\right] e^{j \omega T_{s}}
$$

above expression is a set of first order difference equations. Taking the $\mathrm{Z}$ - transform gives

$$
\begin{aligned}
& z\left\{\hat{x}\left(k T_{s}\right)\right\}=\hat{x}(z) \\
& z\{\hat{x}[(k+1) T]\}=\hat{z x}(z) \\
& z\left\{\hat{v}_{s}\left(k T_{s}\right)\right\}=\hat{v}_{s}(z) \\
& z\left\{\hat{r}_{k}\left(k T_{s}\right)\right\}=\hat{r}_{k}(z)
\end{aligned}
$$

Now in $\mathrm{Z}$ domain we have

$$
\begin{aligned}
\hat{z x}(z)= & \Gamma_{4} \Phi_{4} \Gamma_{3} \Phi_{3} \Gamma_{2} \Phi_{2} \Gamma_{1} \Phi_{1} \hat{x}(z) \\
& +z\left[\left(\Gamma_{4} \beta_{4} \Gamma_{3} \beta_{3} \Gamma_{2} \beta_{2} \Gamma_{1} \eta_{1}+\Gamma_{4} \beta_{4} \Gamma_{3} \beta_{3} \Gamma_{2} \eta_{2}\right.\right. \\
& \left.+\Gamma_{4} \beta_{4} \Gamma_{3} \eta_{3}+\Gamma_{4} \eta_{4} \hat{v_{8}}(z)+\xi_{4} \mu_{4} \hat{r_{4}}(z)\right]
\end{aligned}
$$

In deriving ( $3-17$ ) we made use of the equation $e^{j \omega T_{s}}=z$. By solving the difference equation we find

$$
\begin{aligned}
\hat{x}(z)= & {\left[z I-\Gamma_{4} \Phi_{4} \Gamma_{3} \Phi_{3} \Gamma_{2} \Phi_{2} \Gamma_{1} \Phi_{1}\right]^{-1} } \\
& \cdot z\left[\left(\Gamma_{4} \beta_{4} \Gamma_{3} \beta_{3} \Gamma_{2} \beta_{2} \Gamma_{1} \eta_{1}+\Gamma_{4} \beta_{4} \Gamma_{3} \beta_{3} \Gamma_{2} \eta_{2}\right.\right. \\
& \left.\left.+\Gamma_{4} \beta_{4} \Gamma_{3} \eta_{3}+\Gamma_{4} \eta_{4}\right) \hat{v_{8}}(z)+\xi_{4} \mu_{4} \hat{r_{1}}(z)\right]
\end{aligned}
$$

Since

$$
\begin{aligned}
{\left[z I-\Gamma_{4} \Phi_{4} \Gamma_{3} \Phi_{3} \Gamma_{2} \Phi_{2} \Gamma_{1} \Phi_{1}\right]^{-1}=\left[I-z^{-1} \Gamma_{4} \Phi_{4} \Gamma_{3} \Phi_{3} \Gamma_{2} \Phi_{2} \Gamma_{1} \Phi_{1}\right]^{-1} z^{-1} } \\
=\left[I-e^{-j \omega T_{3}} \Gamma_{4} \Phi_{4} \Gamma_{3} \Phi_{3} \Gamma_{2} \Phi_{2} \Gamma_{1} \Phi_{1}\right]^{-1} z^{-1} \\
=\left[I-\Gamma_{4} \beta_{4} \Gamma_{3} \beta_{3} \Gamma_{2} \beta_{2} \Gamma_{1} \beta_{1}\right]^{-1} z^{-1}
\end{aligned}
$$

In above derivation the definition $\beta_{i}=e^{-j \omega\left(T_{t+1}-T_{i}\right)} \Phi_{i}$, which is given in $(3-6)$, is utilized. Then ( 3 - 18 ) becomes 


$$
\begin{aligned}
\hat{x}(z)= & {\left[I-\Gamma_{4} \beta_{4} \Gamma_{3} \beta_{3} \Gamma_{2} \beta_{2} \Gamma_{1} \beta_{1}\right]^{-1} } \\
& \cdot\left[\left(\Gamma_{4} \beta_{4} \Gamma_{3} \beta_{3} \Gamma_{2} \beta_{2} \Gamma_{1} \eta_{1}+\Gamma_{4} \beta_{4} \Gamma_{3} \beta_{3} \Gamma_{2} \eta_{2}\right.\right. \\
& \left.\left.+\Gamma_{4} \beta_{4} \Gamma_{3} \eta_{3}+\Gamma_{4} \eta_{4}\right) \hat{v_{8}}(z)+\xi_{4} \mu_{4} \hat{r_{1}}(z)\right]
\end{aligned}
$$

The output of the network is $\hat{y}(z)=\hat{C_{1}^{r}} \widehat{x}(z)+E_{1} \hat{v_{s}}(z)$. In the time domain $\hat{x}\left(k T_{s}\right)$ is the state perturbation at the beginning of the first subinterval. The corresponding state space quadruple should be $A_{1}, B_{1}, C_{1}^{T}$ and $E_{1}$. To find the relationship between the control and output, we set $\hat{v}_{z}(z)$ to zero in $(3-20)$. The pulse transfer function of control to output is given by

$$
\frac{\hat{y}(z)}{\hat{r}_{k}(z)}=C_{1}^{T}\left[I-\Gamma_{4} \beta_{4} \Gamma_{3} \beta_{3} \Gamma_{2} \beta_{2} \Gamma_{1} \beta_{1}\right]^{-1} \xi_{4} \mu_{4}
$$

The disturbance signal to output can be found by setting $\hat{r}_{k}(z)$ to zero in $(3-20)$

$$
\begin{aligned}
\frac{\hat{y}(z)}{\hat{v_{s}(z)}}= & C_{1}^{T}\left[I-\Gamma_{4} \beta_{4} \Gamma_{3} \beta_{3} \Gamma_{2} \beta_{2} \Gamma_{1} \beta_{1}\right]^{-1}\left(\Gamma_{4} \beta_{4} \Gamma_{3} \beta_{3} \Gamma_{2} \beta_{2} \Gamma_{1} \eta_{1}\right. \\
& \left.+\Gamma_{4} \beta_{4} \Gamma_{3} \beta_{3} \Gamma_{2} \eta_{2}+\Gamma_{4} \beta_{4} \Gamma_{3} \eta_{3}+\Gamma_{4} \eta_{4}\right)+E_{1}
\end{aligned}
$$

The disadvantage of this method is that the upper limit of non-aliasing frequency range is limited at half the sampling frequency. According to the sampling theorem, the lowest sampling frequency for possible signal reconstruction is two times of the highest frequency component. In applying sampled-data technique once the value of sampling frequency is chosen, the bandwidth of non-aliasing signal reconstruction is decided, which is half of the sampling frequency. 


\section{CHAPTER IV}

\section{THE EXACT SMALLSIGNAL TECHNIQUE}

In this chapter use is made of time-variant system theory to furnish an accurate and general method of frequency analysis of switched networks. This method extends the approach given in [ 1 ] and [ 2 ]. The exact small-signal frequency analysis technique discussed here is based on a state space formulation of circuit equations. A systematic analysis procedure is given which may be applied to any piecewise linear network. The derivations in this chapter are divided into four sections. The first section defines the time varying transfer function for a periodic network. In the second and third section we will introduce its application to switched networks. In the last section a few examples are introduced to verify the results of the application of this method.

\section{THE TIME VARYING TRANSFER FUNCTION}

The following is the definition of the time varying transfer function

$$
H(j \omega, t)=\int_{-\infty}^{\infty} h(t, \tau) e^{-j \omega \tau} d \tau
$$

where $h(t, \tau)$ is an impulse response that gives the response at time $t$ caused by the impulse which was applied $\tau$ time units earlier. This impulse response characterizes the behavior of the system completely, so that for any input $u(t)$, the output can be determined as follows

$$
y(t)=\int_{-\infty}^{\infty} h(t, \tau) u(t-\tau) d \tau
$$

$H(j \omega, t)$ is the Fourier transform of the impulse response $h(t, \tau)$. This expression is similar in form to the convolution integral of linear time-invariant systems. Let us now 
consider an input which has the form $u(t)=e^{j \omega t}$. Substituting this input into ( $4-2$ ) yields

$$
y(t)=\int_{-\infty}^{\infty} h(t, \tau) e^{j \omega(t-\tau)} d \tau=\left(\int_{-\infty}^{\infty} h(t, \tau) e^{-j \omega \tau} d \tau\right) e^{j \omega t}=H(j \omega, t) e^{j \omega t}
$$

From expression ( $4-3$ ) we can see that the output is the product of the time varying transfer function $H(j \omega, t)$, and the input signal $u(t)=e^{j \omega t}$. This gives us a useful definition of $H(j \omega, t)$

$$
H(j \omega, t)=\left(\frac{y(t)}{u(t)}\right)_{u(t)=e^{j \omega t}}
$$

We will make use of ( $4-4)$ in later derivation. In order to find the output frequency response we must find $y(t)$ first. Suppose an input spectrum is given, we can find $u(t)$ by taking the inverse Fourier transform of the input $U(j \omega)$

$$
u(t)=\frac{1}{2 \pi} \int_{-\infty}^{\infty} U(j \omega) e^{j \omega t} d \omega
$$

Substituting ( $4-5)$ in ( $4-2$ ) results in

$$
\begin{aligned}
y(t) & =\int_{-\infty}^{\infty} h(t, \tau)\left[\frac{1}{2 \pi} \int_{-\infty}^{\infty} U(j \omega) e^{j \omega(t-\tau)} d \omega\right] d \tau \\
& =\frac{1}{2 \pi} \int_{-\infty}^{\infty}\left[\int_{-\infty}^{\infty} h(t, \tau) e^{-j \omega \tau} d \tau\right] U(j \omega) e^{j \omega t} d \omega
\end{aligned}
$$

Since it has been defined in $(4-1)$ that

$$
H(j \omega, t)=\int_{-\infty}^{\infty} h(t, \tau) e^{-j \omega \tau} d \tau
$$

the expression ( 4 - 6 ) can be expressed as

$$
y(t)=\frac{1}{2 \pi} \int_{-\infty}^{\infty} H(j \omega, t) U(j \omega) e^{j \omega t} d \omega
$$


Now we can find the output frequency spectrum $Y(j \omega)$. In taking the Fourier transform a new frequency variable is involved. To avoid confusion we use a dummy variable $\mu$ to denote this frequency.

$$
Y(j \mu)=\int_{-\infty}^{\infty} y(t) e^{-j \mu t} d t=\frac{1}{2 \pi} \int_{-\infty}^{\infty}\left[\int_{-\infty}^{-} H(j \omega, t) e^{j \omega t} e^{-j \mu t} d t\right] U(j \omega) d \omega
$$

We may simplify ( $4-9$ ) by assuming that the network is periodic. In this case it can be shown that, for an interval of periodicity of $T_{x}$, the following relation holds

$$
H(j \omega, t)=H\left(j \omega, t+T_{x}\right) \quad \text { for all } t
$$

Given a periodic time varying transfer function we may expand it in a complex Fourier series as follows

$$
H(j \omega, t)=\sum_{n=-\infty}^{\infty} H_{n}(j \omega) e^{j\left(2 \pi / T_{x}\right) n t}
$$

where

$$
H_{n}(j \omega)=\frac{1}{T_{x}} \int_{0}^{T_{x}} H(j \omega, t) e^{-j\left(2 \pi / T_{x}\right) n t} d t
$$

Using ( 4 - 11b ) in ( 4 - 9$)$ gives

$$
\begin{aligned}
Y(j \mu) & =\frac{1}{2 \pi} \int_{-\infty}^{\infty}\left[\int_{-\infty}^{\infty}\left(\sum_{n=-\infty}^{\infty} H_{n}(j \omega) e^{j\left(2 \pi / T_{x}\right) n t}\right) e^{j \omega t} e^{-j \mu t} d t\right] U(j \omega) d \omega \\
& =\frac{1}{2 \pi} \int_{-\infty}^{\infty}\left\{\sum_{n=-\infty}^{\infty} H_{n}(j \omega)\left[\int_{-\infty}^{\infty} e^{j\left(2 n \pi / T_{x}-\mu\right) t} e^{j \omega t} d t\right] U(j \omega)\right\} d \omega \\
& =\frac{1}{2 \pi} \int_{-\infty}^{\infty} 2 \pi\left\{\sum_{n=-\infty}^{\infty} H_{n}(j \omega) \delta\left[\omega-\left(\mu-2 n \pi / T_{x}\right)\right]\right\} U(j \omega) d \omega \\
& =\sum_{n=-\infty}^{\infty} H_{n}\left[j\left(\mu-2 n \pi / T_{x}\right)\right] U\left[j\left(\mu-2 n \pi / T_{x}\right)\right]
\end{aligned}
$$

where $\delta($.$) is Dirac delta function. Since the frequency variable \omega$ has disappeared we can replace the dummy variable $\mu$ with $\omega$ so now we have 
$Y(j \omega)=\sum_{n=-\infty}^{\infty} H_{n}\left[j\left(\omega-2 n \pi / T_{x}\right)\right] U\left[j\left(\omega-2 n \pi / T_{x}\right)\right]$

From ( $4-4)$ we see that $H(j \omega, t)=y(t) e^{-j \omega t}$. Substituting this expression in ( 4 - 11b) gives

$$
H_{n}(j \omega)=\frac{1}{T_{x}} \int_{0}^{T_{x}} y(t) e^{-j \omega t} e^{-j\left(2 \pi T_{x}\right) n t} d t
$$

Refer to ( $4-13$ ) we can have the expression for $U\left[j\left(\omega-2 n \pi / T_{x}\right)\right]$, which is

$$
\begin{aligned}
H_{n}\left[j\left(\omega-2 n \pi / T_{x}\right)\right] & =\frac{1}{T_{x}} \int_{0}^{T_{x}} y(t) e^{-j\left(\omega-2 n \pi / T_{x}\right) t} e^{-j\left(2 \pi / T_{x}\right) n t} d t \\
& =\frac{1}{T_{x}} \int_{0}^{T_{x}} y(t) e^{-j \omega t} d t=\frac{1}{T_{x}} \int_{0}^{T_{x}} H(j \omega, t) d t=H_{0}(j \omega)
\end{aligned}
$$

From the definition ( $4-11 \mathrm{~b}$ ), we see that $H_{0}(j \omega)$ is the DC component of Fourier series of the time varying transfer function $H(j \omega, t)$. It can be obtained by evaluating the average value of $H(j \omega, t)$. Substituting the derived result in ( $4-12)$ yields

$$
Y(j \omega)=\sum_{n=-\infty}^{\infty} H_{0}(j \omega) U\left[j\left(\omega-2 n \pi / T_{x}\right)\right]
$$

$Y(j \omega)$ is thus related to the input spectrum $U(j \omega)$ via the Fourier series expansion of the time varying transfer function $H(j \omega, t)$. We have assumed that $U(j \omega)$ is the Fourier transform of the disturbance. Let's consider this input to output relationship first. In this case the control signal is set to zero. It has been assumed that the input signal has a form of $e^{j \omega t}$. In order to avoid confusion we suppose the frequency of the input signal is $\omega_{0}$. The Fourier transform of $e^{j \omega_{0} t}$ is given by

$$
U(j \omega)=2 \pi \delta\left(\omega-\omega_{0}\right)
$$

Then we have

$$
U\left[j\left(\omega-2 n \pi / T_{x}\right)\right]=2 \pi \delta\left(\omega-\omega_{0}-2 n \pi / T_{x}\right)
$$


Substituting ( $4-18)$ in $(4-16)$ results in

$$
Y(j \omega)=2 \pi \sum_{n=-\infty}^{\infty} H_{0}(j \omega) \delta\left(\omega-\omega_{0}-2 n \pi / T_{x}\right)
$$

Let $\omega_{0}$ take the value of $\omega$, then

$$
Y(j \omega)=2 \pi H_{0}(j \omega)
$$

Next let's consider the relationship of control to output. As discussed earlier, the control signal is proportional to a time quantity. We must find the solution of the differential equation, and express the state variable perturbation as the function of control signal perturbation. However we can just assume that the control signal perturbation also has a form of $e^{j \omega t}$ and that the control input frequency $\omega$ is a multiple $M$ of the system parameter variation frequency $2 \pi / T_{x}$, which means $\omega=2 \pi M / T_{x_{0}}$. Since only control to output response is considered, the source input is held constant so that $u(t)=1$, thus

$$
U(j \omega)=2 \pi \delta(\omega)
$$

Then $(4-16)$ becomes

$$
Y(j \omega)=2 \pi \sum_{n=-\infty}^{\infty} H_{0}(j \omega) \delta\left(\omega-2 n \pi / T_{x}\right)
$$

At any specific frequencies $\omega= \pm 2 \pi M / T_{x}$

$$
Y\left( \pm j 2 \pi M / T_{x}\right)=2 \pi\left[H_{0}\left[ \pm j\left(2 \pi M / T_{x}\right)\right]\right]
$$

Note that since the output is real, so we have

$$
H_{0}\left[+j\left(2 \pi M / T_{x}\right)\right]=H_{0}^{*}\left[-j\left(2 \pi M / T_{x}\right)\right]
$$

where * denotes complex conjugation. If we find $H_{0}(j \omega)$ at a certain frequency value $\omega= \pm 2 \pi M / T_{x}$, we can obtain the output spectrum expression at this value. If $M$ takes a integer value, the output response $Y\left( \pm j 2 \pi M / T_{x}\right)$ can be determined. In fact we can also choose the values of both $M$ and $T_{x}$ to get an arbitrary frequency value at which the 
output frequency spectrum can be determined. In later discussion we will prove it. We can also write an alternative expression for control to output relationship

$$
Y(j \omega)=2 \pi H_{0}(j \omega)
$$

From $(4-20)$ and $(4-23)$ we can see that the two relationships have the same form

$$
Y(j \omega)=2 \pi H_{0}(j \omega)
$$

From the definitions

$$
\begin{aligned}
& H(j \omega, t)=y(t) e^{-j \omega t} \\
& y(t)=C_{i}^{T} x(t)+E_{i} u(t)
\end{aligned}
$$

we can determine the relationship between the frequency response and the state of the system. Once the output spectrum $Y(j \omega)$ is found, $y(t)$ can be calculated by taking inverse Fourier transform of $Y(j \omega) . y(t)$ can be represented in the following form:

$$
\begin{aligned}
& y(t)=A_{0} \cos \left(\omega t+\phi_{0}\right) \\
& \text { where } A_{0}=2\left|H_{0}(j \omega)\right| \\
& \text { and } \phi_{0}=\arg \left[H_{0}(j \omega)\right]
\end{aligned}
$$

Through above procedure we defined a time varying transfer function to describe a linear periodic time varying system, and then derived the output frequency response via Fourier analysis. By using following steps we determined the fundamental Fourier component frequency response of a switching converter:

1) Determine $H(j \omega, t)$ by using ( $4-4)$.

2) Determine $H_{0}(j \omega)$ by using ( $4-15$ ).

3) Draw Bode plot with magnitude response given by $2\left|H_{0}(j \omega)\right|$, and phase response given by angle $\left[H_{0}(j \omega)\right]$.

In next section we will apply the mathematical representation to find an expression of switching converter frequency response. 
THE APPLICATION OF

\section{TIME VARYING TRANSFER FUNCTION}

In last section we found that the time varying transfer function for a piecewise linear network is given by

$$
H(j \omega, t)=y(t) e^{-j \omega t}=\left[C_{i}^{T} x(t)+E_{i} u(t)\right] e^{-j \omega t} t_{i}<t<t_{i+1},\left(i \in 1,2, \ldots, N_{s}\right)(4-28)
$$

It should be pointed out that the time varying transfer function is defined to describe linear systems. To find the linear relationships among all variables we must use small signal modeling technique. The variables of ( $4-28$ ) are instantaneous signals. The small signal perturbations $\hat{t_{i}}, \hat{x_{1}}(t)$ and $\hat{u_{1}(t)}$ are included in these signals. We need to separate each large signal into a steady state operating point and a small signal perturbation, in the same way we did in Chapter II. For example the states of the network can be expressed as

$$
\begin{aligned}
& x\left(t_{i}\right)=X\left(t_{i}\right)+\hat{x}\left(t_{i}\right) \\
& x\left(t_{i+1}\right)=X\left(t_{i+1}\right)+\hat{x}\left(t_{i+1}\right) .
\end{aligned}
$$

Figure 5 depicts the states and switching times for the $i$ th subinterval. From Figure 5 we can see that if there are not any input signals the topology changeover occurs at time $T_{i}$, and the state will be the steady state $X\left(T_{i}\right)$. The expression of the trajectory $X(t)$ in Figure 5 can be derived from ( $2-21$ ) by replacing $T_{i+1}$ with $t$ as follows

$$
\begin{array}{r}
X(t)=e^{A_{(}\left(t-T_{1}\right)} X\left(T_{i}\right)+\left(A_{i}\right)^{-1}\left[e^{A_{(}\left(t-T_{i}\right)}-I\right] B_{i} V_{s} \\
\left(i \in 1,2, \ldots, N_{s}-1\right)
\end{array}
$$

Now by using the results given by $(2-41 a)$ and $(2-41 b)$ we have

$$
\begin{aligned}
& \hat{x}\left(T_{i+1}\right)=\Gamma_{i} \Phi_{i} \hat{x}\left(T_{i}\right)+\Gamma_{i} \eta_{i} \hat{v}_{8}\left(T_{t+1}\right) \quad\left(i \in 1,2, \ldots, N_{s}-1\right) \\
& \hat{x}\left(T_{5}\right)=\Gamma_{4} \Phi_{4} \hat{x}\left(T_{4}\right)+\Gamma_{4} \eta_{4} \hat{v}_{8}\left(T_{5}\right)+\xi_{4} \mu_{4} \hat{r}_{p} e^{j \omega T_{5}}
\end{aligned}
$$

The previously derived time varying transfer function is 


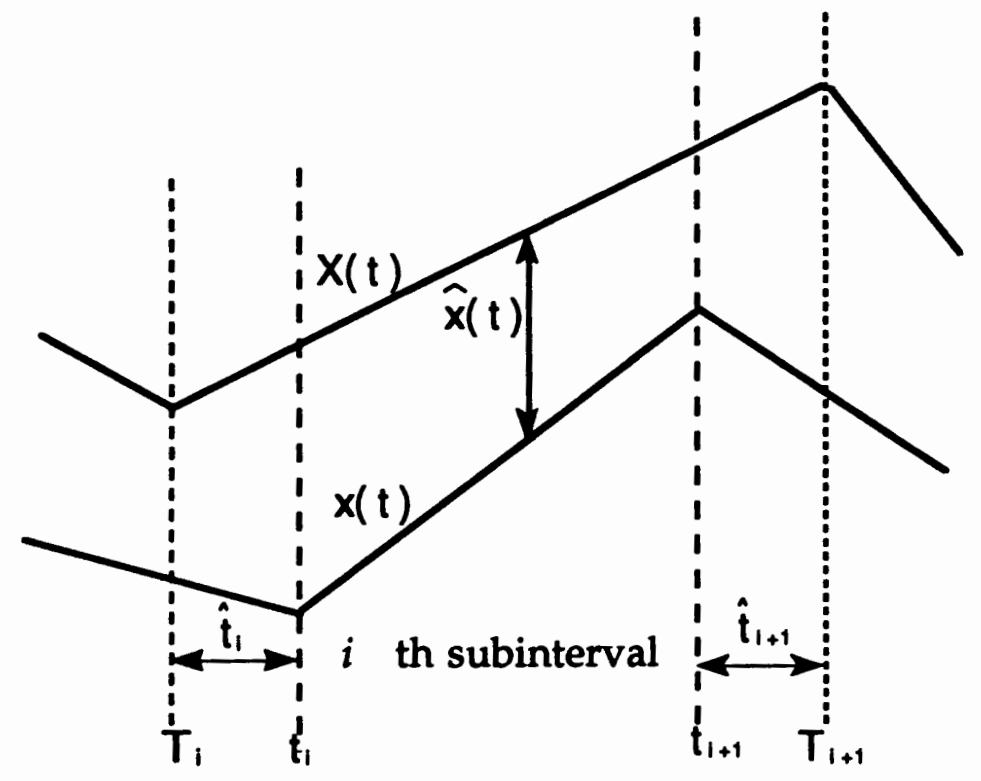

Eigure 5. State and switching times of the $i$ th subinterval. 


$$
H(j \omega, t)=\left[C_{t}^{T} x(t)+E_{t} u(t)\right] e^{-j \omega t}, t_{i}<t<t_{i+1},\left(i \in 1,2, \ldots, N_{s}\right)
$$

Now we need to find the average value of the time varying transfer function over one period of length $T_{x}$. Before doing this we need to determine the length of $T_{x}$ first. There are two frequencies involved in switched network: the control frequency $\omega=2 \pi f$ and the switching frequency $\omega_{s}=2 \pi f_{s}$. We assume that two frequencies are commensurable, that is, both frequencies are an integral multiple of a common frequency $\omega$

$$
\begin{aligned}
& \omega=M \omega_{x} \\
& \omega_{3}=N \omega_{x}
\end{aligned}
$$

Then

$$
T_{x}=2 \pi / \omega_{x}=N T_{s}=M T
$$

where $T_{s}=2 \pi / \omega_{3}$ and $T_{x}=2 \pi / \omega_{x} . T_{x}$ contain $N$ cycles of the switching waveform and $M$ cycles of control waveform. It is the system parameter variation period. Therefore $T_{x}$ is the shortest interval over which the integration is to be performed to calculate $H_{0}(j \omega)$. Figure 6 shows an example of a PWM converter waveform relationship. In this case $T_{x}$ contains eight periods of switching operation, (i. e. $N=8$ ), and one period of control voltage $v_{c}(t)$, (i. e. $M=1$ ) which is one cycle of sinusoidal waveform. The control signal, which is a time perturbation, is proportional to $v_{c}(t)$. The parameter $d_{k}$ is the duty ratio of the $k$-th cycle, and also the control signal of the network. In this case since $N,=2$ the network has only ON and OFF modes. The restriction of the commensurate nature of the relationship between these two frequencies causes no problem as it is possible to choose proper values for $M$ and $N$ to achieve arbitrary precision in attaining a desired control frequency. The value of $\omega_{2}$ is a network parameter, i.e., the switching frequency of the switching device, usually a previously set constant. Since $\omega=M \omega_{x}=(M / N) \omega_{3}$, it is possible to get any input signal frequency value $\omega$ by altering the values of integers $M$ and $N$. This has previously been discussed in the first part of this 


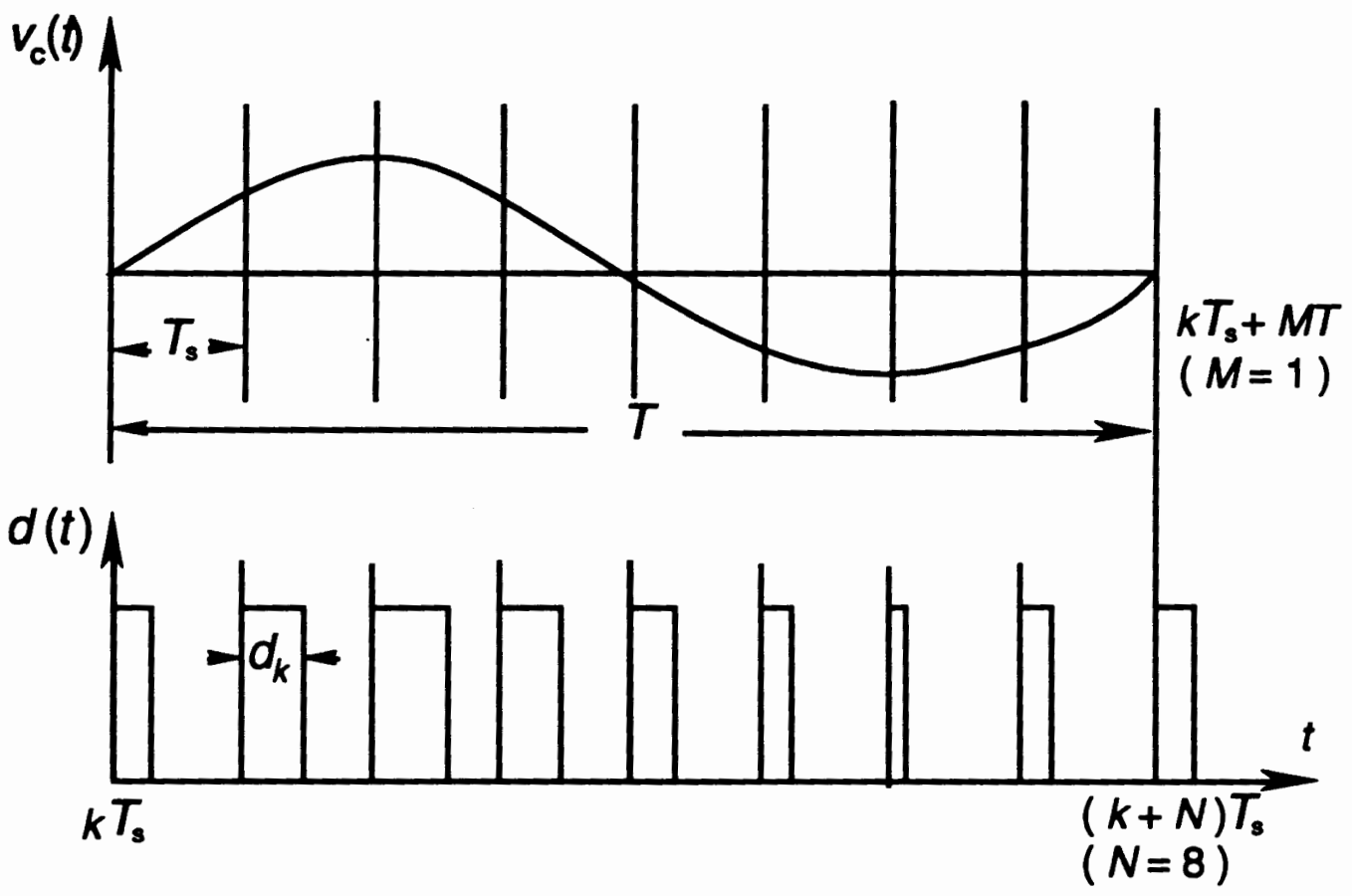

Figure 6. Waveforms showing relationship between the control signal period and switching period. 
chapter. However, since $M$ and $N$ are used to introduce the input signal, whatever values these parameters take does not really affect the dynamic behavior of the network. The relationship between the control voltage and duty ratio $d_{k}$ is also illustrated in Figure 6 .

Now we can find the average value of $H(j \omega, t)$ over the period $T_{x}$

$$
H_{0}(j \omega)=\frac{1}{T_{x}} \int_{0}^{T_{x}} H(j \omega, t) d t
$$

We may separate the period $T_{x}$, into $N$ switching periods,

$T_{x}=N T$,

and each switching period is divided into $N$, subintervals

$T,=\sum_{i=1}^{N}\left(t_{i+1}-t_{i}\right)$

The integration is performed over each subinterval first. Then the sum of integration for all $N$, subinterval is evaluated. For one whole $T_{x}$ period we sum up all the results obtained in each switching cycles. This summation is the time varying transfer function $H(j \omega, t) . H_{0}(j \omega)$ is the average value of $H(j \omega, t)$ and can be expressed as

$$
H_{0}(j \omega)=\frac{1}{N T} \sum_{s=0}^{N-1} \sum_{i=1}^{N_{t}} \int_{t_{i}}^{t_{t+1}}\left[C_{i}^{T} x(t)+E_{i} u(t)\right] e^{-j \omega t} d t
$$

Note that from the definition of the time varying transfer function we have assumed that the input signals have a form $e^{j \omega t}$, so that

$$
H(j \omega, t)=\left(\frac{y(t)}{u(t)}\right)_{u(t)=e^{\alpha a t}}
$$

In our derivation we suppose the input signals have a perturbation magnitude, such as

$$
\begin{aligned}
& \hat{r}(t)=\hat{r}_{p} e^{j \omega t} \\
& \hat{u}(t)=\hat{u} e^{j \omega t}
\end{aligned}
$$

The reason of doing this is that since we are dealing with a linearized small signal model, all those input signal must be small perturbations, and with the perturbation terms as the magnitude for each input signal it will be convenient to identify those first order 
perturbation terms in the states and output terms. In this case we will have to account for appearance of $\hat{r}_{p}$ and $\hat{u}$ in the final results of $H_{0}(j \omega)$. Substituting the instantaneous signal relationship ( 2 - 14) in (4 - 35) and performing the integration results in

$$
\begin{aligned}
H_{0}(j \omega) & =\sum_{k=0}^{N-1} \sum_{i=1}^{N_{s}} \frac{e^{-j \omega t_{i}}}{N T}\left\{C_{i}^{T}\left(j \omega I-A_{i}\right)^{-1}\left[I-e^{-\left(j \omega I-A_{i}\right)\left(t_{t+1}-t_{i}\right)}\right]\right. \\
& +\left[x\left(t_{i}\right)+A_{i}^{-1} B_{i} V_{z}\right]+(1 / j \omega)\left(e^{-j \omega\left(t_{t+1}-t_{i}\right)}-I\right)\left(C_{i}^{T} A_{i}^{-1} B_{i}-E_{i}\right) V_{s} \\
& +e^{j \omega x_{i}}\left[\left[C_{i}^{T}\left(j \omega I-A_{i}\right)^{-1} B_{i}+E_{t}\right]\left(t_{t+1}-t_{i}\right)\right. \\
& \left.\left.+C_{i}^{T}\left(j \omega I-A_{i}\right)^{-2}\left(I-e^{-\left(j \omega I-A_{i}\right)\left(t_{t+1}-t_{i}\right)}\right) B_{i}\right] \hat{u}\right\}
\end{aligned}
$$

Above expression of $H_{0}(j \omega)$ is nonlinear because the input perturbations $\hat{t}_{i}$ and $\vec{t}_{i+1}$, which are included in $t_{i}$ and $t_{i+1}$, in (4-39) appear as exponents. By applying the small signal approximation we can find a linearized system model, which is valid if the perturbations vary in a small range in the vicinity of the steady state operating trajectory. To linearize ( $4-39$ ) we introduce $t_{i}=T_{i}+\hat{t}_{i}, t_{t+1}=T_{i+1}+\hat{t}_{i+1}$ and $x(t)=X(t)+\hat{x}(t)$ in expression ( $4-39)$. Then replacing all those exponents like $e^{\hat{k}}$ with the zeroth and first order, i. e. $1+\hat{k t}$, of its Taylor series, will gives

$$
\begin{aligned}
& H_{0}(j \omega)=\sum_{k=0}^{N \cdot 1} \sum_{i=1}^{N_{s}} \frac{e^{-j \omega T_{i}}}{N T_{s}}\left(I-j \omega \hat{t_{i}}\right)\left\{C_{i}^{T}\left(j \omega t-A_{i}\right)^{-1}\right.
\end{aligned}
$$

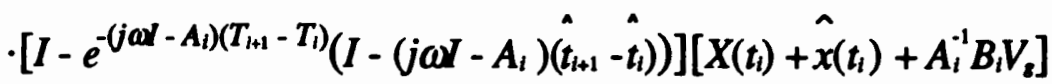

$$
\begin{aligned}
& +(1 / j \omega)\left[e^{-j \omega\left(T_{t+1}-T_{i}\right)}\left[I-j \omega\left(\hat{t}_{i+1}-\hat{t}_{i}\right)\right]-I\right]\left(C_{i}^{T} A_{i}^{-1} B_{i}-E_{i}\right) V_{8} \\
& +e^{j \omega t_{i}}\left(I+j \omega \hat{t}_{i}\right)\left[\left(C_{i}^{T}\left(j \omega I-A_{i}\right)^{-1} B_{i}+E_{i}\right]\left[\left(T_{i+1}-T_{i}\right)+\left(\hat{t}_{i+1}-\hat{t}_{i}\right)\right]\right. \\
& \left.\left.+C_{i}^{T}\left(j \omega I-A_{i}\right)^{-2}\left(I-e^{-\left(j \omega I-A_{i}\right)\left(t_{t+1}-t_{i}\right)}\right) B_{i}\right] \widehat{u}\right\}
\end{aligned}
$$

Now we expand ( $4-40$ ), drop steady state components and neglect the products of perturbation terms. After a lengthy derivation we find a small signal model 


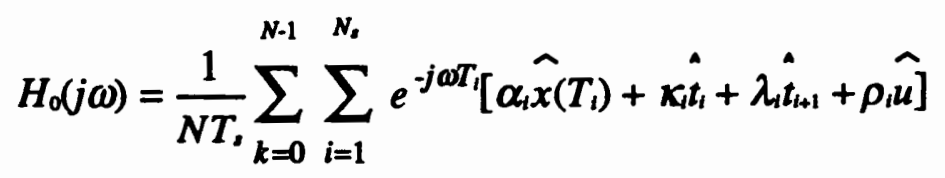

where

and

$$
\begin{aligned}
& \alpha_{i}=C_{i}^{T}\left(j \omega I-A_{i}\right)^{-1}\left[I-e^{-\left(j \omega I-A_{i}\right)\left(T_{i+1}-T_{i}\right)}\right] \\
& \kappa_{i}=-\left[C_{l}^{T} X\left(T_{i}\right)+E_{l} V_{\varepsilon}\right] \\
& \lambda_{i}=\left[C_{i}^{T} X\left(T_{i+1}\right)+E_{l} V_{g}\right] e^{j \omega\left(T_{i+1}-T_{i}\right)} \\
& \rho_{i}=e^{j \omega T_{i}}\left\{C_{i}^{T}\left(j \omega l-A_{i}\right)^{-1}\left[\left(T_{i+1}-T_{i}\right) B_{i}-\eta_{i}\right]+E_{i}\left(T_{i+1}-T_{i}\right)\right\}
\end{aligned}
$$

where

$$
\eta_{i}=\left(j \omega I-A_{i}\right)\left[I-e^{-\left(j \omega t-A_{i}\right)\left(T_{t+1}-T_{i}\right)}\right] B_{i}
$$

Above expression for the transfer function $H_{0}(j \omega)$ can be applied to analyze any piecewise linear network. In the next section we will use this result to find the transfer functions for a generalized switched network.

\section{THE DERIVATION OF TRANSFER FUNCTIONS BY USING THE EXACT SMALL-SIGNAL TECHNIQUE}

In this section we will apply the exact technique to analyze the same switched network which was analyzed by using the sampled-data representation method in Chapter III. The object is to find two "general" transfer functions for a large category of switching converters, that the number of topologies they may take can be any positive integer $N_{s}$. First let's consider a case of $N_{s}=4$. Then ( $4-41$ ) becomes

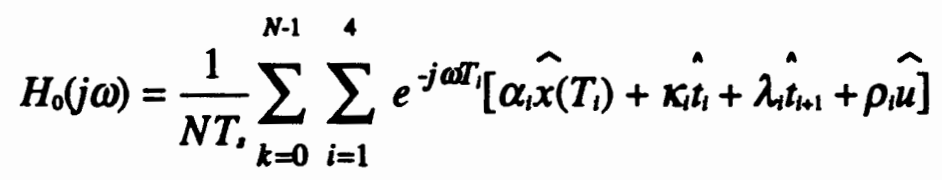

Along with the state and disturbance signal perturbations there are intermediate variables $\hat{t}_{i}$ and $\hat{t}_{i+1}$. The control input signal constitutes one or more of these time perturbations. That is to say that these time perturbations may be explicitly described in terms of other 
variables. Our first step is to evaluate all time perturbation terms which are included in the summation

$$
\sum_{i=1}^{4} e^{-j \omega T_{i}}\left(\kappa_{i} \hat{t}_{i}+\lambda_{i} \hat{t_{i+1}}\right)
$$

Expression ( 4 - 48 ) is a component of the summation in ( $4-47$ ). We expand the sum ( 4 - 48 ) so that it appear as follows

$$
\begin{aligned}
& \sum_{i=1}^{4} e^{-j \omega T_{1}}\left(\kappa_{i} \dot{t_{i}}+\lambda_{i} \hat{t}_{i+1}\right) \\
& =\left[e^{-j \omega T_{1}} \kappa_{i} \hat{t}_{1}+e^{-j \omega T_{4}} \lambda_{4} \hat{t}_{5}\right]+\sum_{i=1}^{3}\left(e^{-j \omega T_{i}} \lambda_{i}+e^{-j \omega T_{i+1}} \kappa_{i+1}\right) \hat{t}_{i+1}
\end{aligned}
$$

The first two terms of ( 4 - 49 ) can be expressed as

$$
\begin{aligned}
e^{-j \omega T_{1}} \hat{K}_{1} \hat{t}_{1}+e^{-j \omega T_{4}} \lambda_{4_{5}} & =e^{-j \omega T_{1}}\left\{-\left[C_{1}^{T} X\left(T_{1}\right)+E_{1} V_{8}\right]\right\} \hat{t_{1}} \\
& +e^{-j \omega T_{4}}\left[C_{4}^{T} X\left(T_{5}\right)+E_{4} V_{8}\right] e^{-j \omega\left(T_{5}-T_{4}\right)_{t_{5}}}
\end{aligned}
$$

Since $N_{s}=4$ the following relations hold

$$
C_{1}^{T}=C_{5}^{T}, \quad E_{2}=E_{s}, \quad X\left(T_{1}\right)=X\left(T_{3}\right) \quad \text { and } \quad \dot{t}_{1}=\dot{t}_{s} e^{-j \omega\left(T_{3}-T_{1}\right)}
$$

Then

$$
\begin{aligned}
& e^{-j \omega T_{1}} \hat{K}_{1} \hat{t}_{1}+e^{-j \omega T_{4}} \hat{\lambda}_{4} \hat{t}_{5} \\
& \quad=e^{-j \omega T_{5}}\left[\left(C_{4}^{T}-C_{s}^{T}\right) X\left(T_{3}\right)+\left(E_{4}-E_{3}\right) V_{8}\right] \hat{t}_{5}
\end{aligned}
$$

We define

$$
\zeta_{i}=\left(C_{l}^{T}-C_{i+1}^{T}\right) X\left(T_{i+1}\right)+\left(E_{i}-E_{i+1}\right) V_{8}
$$

such that

$$
e^{-j \omega T_{1}} \hat{K}_{1} \hat{t}_{1}+e^{-j \omega T_{A}} \lambda_{A_{s}} \hat{t}_{5}=e^{-j \omega T_{s}} \hat{G}_{t_{5}}
$$

The second part of ( 4 - 48 ) can be evaluated as

$$
\sum_{i=1}^{3} e^{-j \omega T_{1}}\left(\kappa_{i} \hat{t}_{i}+\lambda_{i} \hat{t}_{i+1}\right)
$$




$$
\begin{aligned}
& =\sum_{i=1}^{3}\left\{e^{-j \omega T_{i}}\left[C_{i}^{T} X\left(T_{i+1}\right)+E_{i} V_{8}\right] e^{-j \omega\left(T_{i+1}-T_{i}\right)}\right. \\
& \left.+e^{-j \omega T_{i+1}}\left[-\left(C_{i+2}^{T} X\left(T_{i+1}\right)+E_{i+1} V_{8}\right)\right]\right\} \hat{t}_{i+1} \\
& =\sum_{i=1}^{3} e^{-j \omega T_{i+1}}\left[\left(C_{i}^{T}-C_{i+1}^{T}\right) X\left(T_{i+1}\right)+\left(E_{l}-E_{i+1}\right) V_{8}\right] \hat{t}_{i+1} \\
& =\sum_{i=1}^{3} e^{-j \omega T_{i+1}} \hat{\zeta}_{i t+1}
\end{aligned}
$$

The expression ( 4 - 48 ) can be expressed as

$$
\sum_{i=1}^{4} e^{-j \omega T_{i}}\left(\kappa_{i} \hat{t}_{i}+\lambda_{i} \hat{t}_{i+1}\right)=\sum_{i=1}^{4} e^{-j \omega T_{i+1}} \hat{\zeta}_{i t+1}
$$

Now the expression( $4-47$ ) becomes

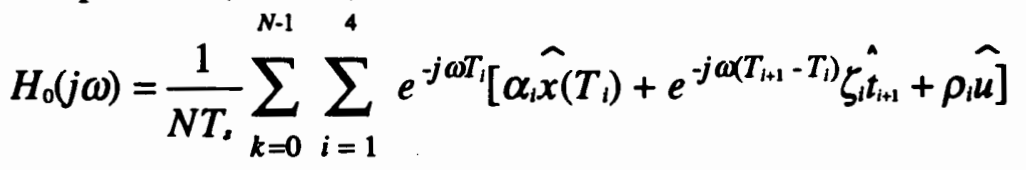

The perturbation terms of $t_{i}$ in ( $4-47$ ) has disappeared. To evaluate those terms with $\hat{t}_{t+1}$ we can use the results presented in $(2-39 a)$ and $(2-39 b)$ of Chapter II for the case $N_{s}=4$

$$
\begin{aligned}
& \hat{t_{i+1}}=-\hat{h}_{i}^{T} \mu_{i} \Phi_{i} \hat{x}\left(T_{i}\right)-\hat{h}_{i}^{T} \mu_{i} \hat{\eta}_{i} \hat{v}_{8}\left(T_{i+1}\right), \quad(i \in 1,2,3) \\
& \hat{t_{5}}=\mu_{4} \hat{r}_{k}\left(T_{5}\right)-\hat{h}_{4}^{T} \mu_{4} \Phi_{4} \hat{x}\left(T_{4}\right)-\hat{h}_{4}^{T} \mu_{4} \hat{\eta}_{4} \hat{u} e^{j \omega T_{5}}
\end{aligned}
$$

In ( $4-56)$ the equation $\hat{v_{8}}\left(T_{i+1}\right)=\hat{u} e^{j \omega T_{i+1}}$ is used. Substituting $(4-56 \mathrm{a})$ and $(4-56 b)$ in $(4-55)$ produces

$$
\begin{aligned}
H_{0}(j \omega) & =\frac{1}{N T} \sum_{k=0}^{N-1}\left\{\sum _ { i = 1 } ^ { 4 } \left[e^{-j \omega T_{i}}\left(\alpha_{i}-\zeta_{i} h_{i}{ }^{T} \mu_{i} \beta_{i}\right) \hat{x}\left(T_{i}\right)\right.\right. \\
& \left.\left.+\left(e^{-j \omega T_{i}} \rho_{i}-\zeta_{i} h_{i}{ }^{T} \mu_{4} h_{i}\right) \hat{u}\right]+\zeta_{4} \hat{\mu}_{4} \hat{r}_{p}\right\}
\end{aligned}
$$

In above derivation the following some previously obtained results are used

$$
e^{-j \omega T_{l+1}} \Phi_{i}=e^{-j \omega T_{i}} \beta_{i}
$$




$$
\left.\zeta_{4} \mu_{4} e^{-j \omega T_{s_{k}}^{\hat{r}}} \hat{r}_{s}\right)=\zeta_{4} \mu_{4} e^{-j \omega T_{r_{p}}} e^{j \omega T_{s}}=\zeta_{4} \mu_{4} \hat{r_{p}}
$$

In expression $(4-57)$ there is no intermediate time variable $\hat{t}_{t+1}$. Instead the perturbation of control input signal appears. All we need to do is evaluate state perturbations $\hat{x}\left(T_{1}\right),(i \in 1,2,3,4)$. In doing so we can also use the results of ( 2 - 41a ) and ( 2-41b) in Chapter II, for the case $N_{s}=4$

$$
\begin{aligned}
& \hat{x}\left(T_{i+1}\right)=\Gamma_{i} \hat{\Phi_{2}} \hat{x}\left(T_{i}\right)+\Gamma_{i} \eta_{i v_{8}}\left(T_{i+1}\right), \quad(i \in 1,2,3) \\
& \hat{x}\left(T_{5}\right)=\Gamma_{4} \Phi_{4} \hat{x}\left(T_{4}\right)+\Gamma_{4} \eta_{4} \hat{v}_{8}\left(T_{5}\right)+\xi_{4} \mu_{4} \hat{r_{p}} e^{j \omega T_{5}}
\end{aligned}
$$

Then the state perturbation $\hat{x}\left(T_{i}\right)(i \in 2,3,4,5)$ can be evaluated in terms of the initial state perturbation $\hat{x}\left(T_{1}\right)$, input signal perturbations $\hat{r}_{p}$ and $\hat{v}_{s}(T)(i \in 2,3,4,5)$

$$
\begin{aligned}
& \hat{x}\left(T_{2}\right)=\Gamma_{1} \Phi_{1} \hat{x}\left(T_{1}\right)+\Gamma_{1} \hat{\eta}_{1} \hat{u} e^{j \omega T_{2}} \\
& =\Gamma_{1} \beta_{1} e^{j \omega\left(T_{2}-T_{1}\right)} \hat{x\left(T_{1}\right)}+\Gamma_{1} \hat{\eta}_{1} \hat{u} e^{j \omega T_{2}} \\
& \hat{x}\left(T_{3}\right)=\Gamma_{2} \Phi_{2} \Gamma_{1} \Phi_{1} \hat{x}\left(T_{1}\right)+\Gamma_{2} \Phi_{2} \Gamma_{1} \eta_{1} \hat{u} e^{j \omega T_{2}}+\Gamma_{2} \eta_{2} \hat{u} e^{j \omega T_{3}} \\
& =\Gamma_{2} \beta_{2} \Gamma_{1} \beta_{1} e^{j \omega\left(T_{3}-T_{1}\right)} \hat{x}\left(T_{1}\right)+\left(\Gamma_{2} \beta_{2} \Gamma_{1} \eta_{1}+\Gamma_{2} \eta_{2}\right) \hat{u} e^{j \omega T_{3}} \\
& \hat{x}\left(T_{4}\right)=\Gamma_{3} \Phi_{3} \Gamma_{2} \Phi_{2} \Gamma_{1} \Phi_{1} \hat{x}\left(T_{1}\right)+\Gamma_{3} \Phi_{3} \Gamma_{2} \Phi_{2} \Gamma_{1} \hat{\eta}_{1} \hat{u}{ }^{j \omega T_{2}}+\Gamma_{3} \Phi_{3} \Gamma_{2} \eta_{2} \hat{u} e^{j \omega T_{3}}+\Gamma_{3} \hat{\eta}_{3} \hat{u} e^{j \omega T_{4}} \\
& =\Gamma_{3} \beta_{3} \Gamma_{2} \beta_{2} \Gamma_{1} \beta_{1} e^{j \omega\left(T_{4}-T_{1}\right)} \hat{x}\left(T_{1}\right) \\
& +\left(\Gamma_{3} \beta_{3} \Gamma_{2} \beta_{2} \Gamma_{1} \eta_{1}+\Gamma_{3} \beta_{3} \Gamma_{2} \eta_{2}+\Gamma_{3} \eta_{3}\right) \hat{u} j \omega T_{4}
\end{aligned}
$$

We also need to find $\hat{x}\left(T_{5}\right)$

$$
\begin{aligned}
& \hat{x}\left(T_{5}\right)=\Gamma_{4} \Phi_{4} \Gamma_{3} \Phi_{3} \Gamma_{2} \Phi_{2} \Gamma_{1} \hat{\Phi_{1}} \hat{x}\left(T_{1}\right)+\Gamma_{4} \Phi_{4} \Gamma_{3} \Phi_{3} \Gamma_{2} \Phi_{2} \Gamma_{1} \hat{\eta_{1}} \hat{u} e^{j \omega T_{2}} \\
& +\Gamma_{4} \Phi_{4} \Gamma_{3} \Phi_{3} \Gamma_{2} \eta_{2} \hat{u} e^{j \omega T_{3}}+\Gamma_{4} \Phi_{4} \Gamma_{3} \eta_{3} \hat{e^{j \omega}}+\Gamma_{4} \eta_{4} \hat{u}{ }^{j \omega T_{5}}+\xi_{4} \mu_{4} \hat{r_{2}} e^{j \omega T_{5}} \\
& =\Gamma_{4} \beta_{4} \Gamma_{3} \beta_{3} \Gamma_{2} \beta_{2} \Gamma_{1} \beta_{1} e^{j \omega\left(T_{5}-T_{1}\right)} \hat{x}\left(T_{1}\right)+\left(\Gamma_{4} \beta_{4} \Gamma_{3} \beta_{3} \Gamma_{2} \beta_{2} \Gamma_{1} \eta_{1}\right. \\
& \left.+\Gamma_{4} \beta_{4} \Gamma_{3} \beta_{3} \Gamma_{2} \eta_{2}+\Gamma_{4} \beta_{4} \Gamma_{3} \eta_{3}+\Gamma_{4} \eta_{4}\right) \hat{u} e^{j \omega T_{5}}+\xi_{4} \mu_{4} \hat{r}_{p} e^{j \omega T_{5}}
\end{aligned}
$$


40

The equation $e^{-j \omega T_{t+1}} \Phi_{i}=e^{-j \omega T_{1}} \beta_{i}$ is used in above derivation. In expression( 4 - 57 ) pick the terms with $\hat{x}\left(T_{i}\right)$, which is the summation

$$
\begin{aligned}
\sum_{i=1}^{4} e^{-j \omega T_{1}}\left(\alpha_{i}-\zeta_{1} h_{i}^{T} \mu_{i} \beta_{i}\right) \hat{x}\left(T_{1}\right) & \\
= & e^{-j \omega T_{1}}\left(\alpha_{1}-\zeta_{1} h_{1}^{T} \mu_{1} \beta_{1}\right) \hat{x}\left(T_{1}\right)+e^{-j \omega T_{2}}\left(\alpha_{2}-\zeta_{2} h_{2}^{T} \mu_{2} \beta_{2}\right) \hat{x}\left(T_{2}\right) \\
& =e^{-j \omega T_{3}}\left(\alpha_{3}-\zeta_{3} h_{3}^{T} \mu_{3} \beta_{3}\right) \hat{x}\left(T_{3}\right)+e^{-j \omega T_{4}}\left(\alpha_{4}-\zeta_{4} h_{4}^{T} \mu_{4} \beta_{4}\right) \hat{x}\left(T_{4}\right)
\end{aligned}
$$

The terms with $\hat{x}\left(T_{2}\right), \hat{x}\left(T_{3}\right)$ and $\hat{x}\left(T_{4}\right)$ in $(4-66)$ can be written as

$$
\begin{aligned}
& e^{-j \omega T_{2}}\left(\alpha_{2}-\zeta_{2} h_{2}{ }^{T} \mu_{2} \beta_{2}\right) \hat{x}\left(T_{2}\right) \\
& =e^{-j \omega T_{2}}\left(\alpha_{2}-\zeta_{2} h_{2}{ }^{T} \mu_{2} \beta_{2}\right)\left[\Gamma_{1} \beta_{1} e^{j \omega\left(T_{2}-T_{1}\right)} \hat{x}\left(T_{1}\right)+\Gamma_{1} \hat{\eta}_{1} \hat{u e^{j \omega T_{2}}}\right] \\
& =e^{-j \omega T_{1}}\left(\alpha_{2} \Gamma_{1} \beta_{1}-\zeta_{2} h_{2}^{T} \mu_{2} \beta_{2} \Gamma_{1} \beta_{1}\right) \hat{x}\left(T_{1}\right)+\left(\alpha_{2} \Gamma_{1} \eta_{1}+\zeta_{2} h_{2}^{T} \mu_{2} \beta_{2} \Gamma_{1} \eta_{1}\right) \hat{u} \\
& e^{-j \omega T_{3}}\left(\alpha_{3}-\zeta_{3} h_{3}^{T} \mu_{3} \beta_{3}\right) \hat{x}\left(T_{3}\right) \\
& =e^{-j \omega T_{3}}\left(\alpha_{3}-\zeta_{3} h_{3}^{T} \mu_{3} \beta_{3}\right)\left[\Gamma_{2} \beta_{2} \Gamma_{1} \beta_{1} e^{j \omega\left(T_{3}-T_{1}\right)} \hat{x}\left(T_{1}\right)+\left(\Gamma_{2} \beta_{2} \Gamma_{1} \eta_{1}+\Gamma_{2} \eta_{2}\right) \hat{u} e^{j \omega T_{3}}\right] \\
& =e^{-j \omega T_{1}}\left(\alpha_{3} \Gamma_{2} \beta_{2} \Gamma_{1} \beta_{1}-\zeta_{3} h_{3}^{T} \mu_{3} \beta_{3} \Gamma_{2} \beta_{2} \Gamma_{1} \beta_{1}\right) \hat{x}\left(T_{1}\right)+\left(\alpha_{3} \Gamma_{2} \beta_{2} \Gamma_{1} \eta_{1}+\alpha_{3} \Gamma_{2} \eta_{2}\right. \\
& \left.-\zeta_{3} h_{3}^{T} \mu_{3} \beta_{3} \Gamma_{2} \beta_{2} \Gamma_{1} \eta_{1}-\zeta_{3} h_{3}^{T} \mu_{3} \beta_{3} \Gamma_{2} \eta_{2}\right) \hat{u} \\
& e^{-j \omega T_{4}}\left(\alpha_{4}-\zeta_{4} h_{4}^{T} \mu_{4} \beta_{4}\right) \hat{x}\left(T_{4}\right) \\
& =e^{-j \omega T_{4}}\left(\alpha_{4}-\zeta h_{4}{ }^{T} \mu_{4} \beta_{4}\right)\left[\Gamma_{3} \beta_{3} \Gamma_{2} \beta_{2} \Gamma_{1} \beta_{1} e^{j \omega\left(T_{4}-T_{1}\right)} \hat{x}\left(T_{1}\right)\right. \\
& +\left(\Gamma_{3} \beta_{3} \Gamma_{2} \beta_{2} \Gamma_{1} \eta_{1}+\Gamma_{3} \beta_{3} \Gamma_{2} \eta_{2}+\Gamma_{3} \eta_{3}\right) \hat{\left.u e^{j \omega T_{4}}\right]} \\
& =e^{-j \omega T_{1}}\left(\alpha_{4} \Gamma_{3} \beta_{3} \Gamma_{2} \beta_{2} \Gamma_{1} \beta_{1}-\zeta_{4} h_{4}^{T} \mu_{4} \beta_{4} \Gamma_{4} \beta_{3} \Gamma_{2} \beta_{2} \Gamma_{1} \beta_{1}\right) \hat{x}\left(T_{1}\right) \\
& +\left(\alpha_{4} \Gamma_{3} \eta_{3}+\alpha_{4} \Gamma_{3} \beta_{3} \Gamma_{2} \eta_{2}+\alpha_{4} \Gamma_{3} \beta_{3} \Gamma_{2} \beta_{2} \Gamma_{1} \eta_{1}-\zeta_{4} h_{4}^{T} \mu_{4} \beta_{4} \Gamma_{3} \eta_{3}\right. \\
& \left.-\zeta_{4} h_{4}^{T} \mu_{4} \beta_{4} \Gamma_{3} \beta_{3} \Gamma_{2} \eta_{2}-\zeta_{4} h_{4}^{T} \mu_{4} \beta_{4} \Gamma_{3} \beta_{3} \Gamma_{2} \beta_{2} \Gamma_{1} \eta_{1}\right) \hat{u}
\end{aligned}
$$


The summation ( 4 - 66 ) can be evaluated as

$$
\begin{aligned}
& \sum_{i=1}^{4} e^{-j \omega T_{1}}\left(\alpha_{1}-\zeta_{i} h_{i}^{T} \mu_{i} \beta_{i}\right) \hat{x}\left(T_{i}\right) \\
&= e^{-j \omega T_{1}}\left[\alpha_{4}+\left(\alpha_{2} \Gamma_{1}-\zeta_{1} h_{1}^{T} \mu_{1}\right) \beta_{1}+\left(\alpha_{3} \Gamma_{2}-\zeta_{2} h_{2}^{T} \mu_{2}\right) \beta_{2} \Gamma_{1} \beta_{1}+\left(\alpha_{4} \Gamma_{3}\right.\right. \\
&\left.\left.-\zeta_{3} h_{3}^{T} \mu_{3}\right) \beta_{3} \Gamma_{2} \beta_{2} \Gamma_{1} \beta_{1}-\zeta_{4} h_{4}^{T} \mu_{4} \beta_{4} \Gamma_{3} \beta_{3} \Gamma_{2} \beta_{2} \Gamma_{1} \beta_{1}\right] \hat{x}\left(T_{1}\right) \\
&+\left[\left(\alpha_{2}-\zeta_{2} h_{2}^{T} \mu_{2} \beta_{2}\right) \Gamma_{1} \eta_{1}+\left(\alpha_{3}-\zeta_{3} h_{3}^{T} \mu_{3} \beta_{3}\right)\left(\Gamma_{2} \beta_{2} \Gamma_{1} \eta_{1}+\Gamma_{2} \eta_{2}\right)+\left(\alpha_{4}-\zeta 4 h_{4}^{T} \mu_{4} \beta_{4}\right)\right. \\
&\left..\left(\Gamma_{3} \beta_{3} \Gamma_{2} \beta_{2} \Gamma_{1} \eta_{1}+\Gamma_{3} \beta_{3} \Gamma_{2} \eta_{2}+\Gamma_{3} \eta_{3}\right)\right] \hat{u}+\zeta_{4} \mu_{4} \hat{r}_{p}
\end{aligned}
$$

Note that in $(4-70)$ all those terms dealing with $\widehat{x}\left(T_{i}\right)$ have been replaced by the terms of state $\hat{x}\left(T_{1}\right), \hat{r}_{p}$ and $\hat{u}$. The term of $\hat{x}\left(T_{1}\right)$ can be evaluated by solving the difference equation given by expression ( $3-20$ ). The difference equation is given by

$$
\begin{aligned}
\hat{x}\left(T_{5}\right)= & \Gamma_{4} \beta_{4} \Gamma_{3} \beta_{3} \Gamma_{2} \beta_{2} \Gamma_{1} \beta_{1} e^{j \omega \alpha\left(T_{3}-T_{1}\right)} \hat{x}\left(T_{1}\right)+\left(\Gamma_{4} \beta_{4} \Gamma_{3} \beta_{3} \Gamma_{2} \beta_{2} \Gamma_{1} \eta_{1}+\Gamma_{4} \beta_{4} \Gamma_{3} \beta_{3} \Gamma_{2} \eta_{2}\right. \\
& \left.+\Gamma_{4} \beta_{4} \Gamma_{3} \eta_{3}+\Gamma_{4} \eta_{4}\right) \hat{u e^{j \omega T_{5}}}+\xi_{4} \hat{r}_{4} \hat{r}_{p} e^{j \omega T_{5}}
\end{aligned}
$$

Let $\hat{x}\left(T_{1}\right)=\hat{x}(k T)$ and $\hat{x}\left(T_{s}\right)=\hat{x}\left[(k+1) T_{\text {, }}\right.$. Then

$$
\begin{gathered}
\hat{x}\left[(k+1) T_{3}\right]=\Gamma_{4} \beta_{4} \Gamma_{3} \beta_{3} \Gamma_{2} \beta_{2} \Gamma_{1} \beta_{1} e^{j \omega\left(T_{5}-T_{1}\right)} \hat{x}\left(k T_{3}\right)+\left(\Gamma_{4} \beta_{4} \Gamma_{3} \beta_{3} \Gamma_{2} \beta_{2} \Gamma_{1} \eta_{1}\right. \\
\left.+\Gamma_{4} \beta_{4} \Gamma_{3} \beta_{3} \Gamma_{2} \eta_{2}+\Gamma_{4} \beta_{4} \Gamma_{3} \eta_{3}+\Gamma_{4} \eta_{4}\right) \hat{u} e^{j \omega T_{3}}+\xi_{4} \mu_{4} \hat{r}_{p} e^{j \omega T_{3}}
\end{gathered}
$$

The steady state solution of the difference equation is

$$
\begin{aligned}
\hat{x}\left(k T_{1}\right) & =e^{j \omega k T_{s}}\left\{( I - \Gamma _ { 4 } \beta _ { 4 } \Gamma _ { 3 } \beta _ { 3 } \Gamma _ { 2 } \beta _ { 2 } \Gamma _ { 1 } \beta _ { 4 } ) ^ { - 1 } \left[\left(\Gamma_{4} \beta_{4} \Gamma_{3} \beta_{3} \Gamma_{2} \beta_{2} \Gamma_{1} \eta_{1}\right.\right.\right. \\
& \left.\left.\left.+\Gamma_{4} \beta_{4} \Gamma_{3} \beta_{3} \Gamma_{2} \eta_{2}+\Gamma_{4} \beta_{4} \Gamma_{3} \eta_{3}+\Gamma_{4} \eta_{4}\right) \hat{u}+\xi_{4} \mu_{4} \hat{r_{p}}\right]\right\}
\end{aligned}
$$

Now the term of $\hat{x}\left(T_{1}\right)$ is expressed in terms of $\hat{r}_{p}$ and $\hat{u}$. The next step is to evaluate the second sum term in ( 4 - 57)

$$
\sum_{i=1}^{4}\left(e^{-j \omega T_{i}} \rho_{i}-\zeta h_{i}{ }^{T} \mu_{i} \eta_{i}\right) \hat{u}
$$


The expression for $\rho_{t}$ is given by $(4-45)$

$$
\rho_{i}=e^{j \omega T_{i}}\left\{C_{i}^{T}\left(j \omega I-A_{i}\right)^{-1}\left[\left(T_{i+1}-T_{i}\right) B_{i}-\eta_{i}\right]+E_{i}\left(T_{i+1}-T_{i}\right)\right\}
$$

Then

$$
\begin{aligned}
\sum_{i=1}^{4}\left(e^{-j \omega T_{i}}\right. & \left.\rho_{i}-\zeta h_{i}^{T} \mu_{1} \eta_{1}\right) \hat{u} \\
& =\sum_{i=1}^{4}\left\{\left(C_{i}^{T}\left(j \omega I-A_{1}\right)^{-1}\left[\left(T_{i+1}-T_{i}\right) B_{i}-\eta_{i}\right]+E_{i}\left(T_{i+1}-T_{i}\right)\right)\right. \\
& \left.-\left(\zeta_{1} h_{1}^{T} \mu_{1} \eta_{1}+\zeta_{2} h_{2}^{T} \mu_{2} \eta_{2}+\zeta_{3} h_{3}^{T} \mu_{3} \eta_{3}+\zeta_{4} h_{4}^{T} \mu_{4} \eta_{4}\right)\right\} \hat{u}
\end{aligned}
$$

Then expression ( 4 - 58 ) becomes

$$
\begin{aligned}
H_{0}(j \omega) & =\frac{1}{T_{3}}\left(\left\{\left[\alpha_{1}+\left(\alpha_{2} \Gamma_{1}-\zeta_{1} h_{1}^{T} \mu_{1}\right) \beta_{1}+\left(\alpha_{3} \Gamma_{2}-\zeta_{2} h_{2}^{T} \mu_{2}\right) \beta_{2} \Gamma_{1} \beta_{1}\right.\right.\right. \\
& \left.+\left(\alpha_{4} \Gamma_{3}-\zeta_{3} h_{3}^{T} \mu_{3}\right) \beta_{3} \Gamma_{2} \beta_{2} \Gamma_{1} \beta_{1}-\zeta_{4} h_{4}^{T} \mu_{4} \beta_{4} \Gamma_{3} \beta_{3} \Gamma_{2} \beta_{2} \Gamma_{1} \beta_{1}\right] \\
& \left.\cdot\left(I-\Gamma_{4} \beta_{4} \Gamma_{3} \beta_{3} \Gamma_{2} \beta_{2} \Gamma_{1} \beta_{1}\right)^{-1} \xi_{4} \mu_{4}+\zeta_{4} \mu_{4}\right\}_{p_{p}} \\
& +\left(\left[\alpha_{1}+\left(\alpha_{2} \Gamma_{1}-\zeta_{1} h_{1}^{T} \mu_{1}\right) \beta_{1}+\left(\alpha_{3} \Gamma_{2}-\zeta_{2} h_{2}^{T} \mu_{2}\right) \beta_{2} \Gamma_{1} \beta_{1}\right.\right. \\
& \left.+\left(\alpha_{4} \Gamma_{3}-\zeta_{3} h_{3}^{T} \mu_{3}\right) \beta_{3} \Gamma_{2} \beta_{2} \Gamma_{1} \beta_{1}-\zeta_{4} h_{4}^{T} \mu_{4} \beta_{4} \Gamma_{3} \beta_{3} \Gamma_{2} \beta_{2} \Gamma_{1} \beta_{1}\right] \\
& \left.\cdot\left(I-\Gamma_{4} \beta_{4} \Gamma_{3} \beta_{3} \Gamma_{2} \beta_{2} \Gamma_{1} \beta_{1}\right)^{-1} \Gamma_{4}-\zeta_{4} h_{4}^{T} \mu_{4}\right) \\
& +\left(\beta_{4} \Gamma_{3} \beta_{3} \Gamma_{2} \beta_{2} \Gamma_{1} \eta_{1}+\beta_{4} \Gamma_{3} \beta_{3} \Gamma_{2} \eta_{2}+\beta_{4} \Gamma_{3} \eta_{3}+\eta_{4}\right) \\
& +\left(\alpha_{2} \Gamma_{1}-\zeta_{1} h_{1}^{T} \mu_{1}\right) \eta_{1}+\left(\alpha_{3} \Gamma_{2}-\zeta_{2} h_{2}^{T} \mu_{2}\right)\left(\beta_{2} \Gamma_{1} \eta_{1}+\eta_{2}\right) \\
& +\left(\alpha_{4} \Gamma_{3}-\zeta_{3} h_{3}^{T} \mu_{3}\right)\left(\beta_{3} \Gamma_{2} \beta_{2} \Gamma_{1} \eta_{1}+\beta_{3} \Gamma_{2} \eta_{2}+\eta_{3}\right) \\
& +C_{1}^{T}\left(j \omega I-A_{1}\right)^{-1}\left[\left(T_{2}-T_{1}\right) B_{1}-\eta_{1}\right]+E_{1}\left(T_{2}-T_{1}\right) \\
& +C_{2}^{T}\left(j \omega I-A_{2}\right)^{-1}\left[\left(T_{3}-T_{2}\right) B_{2}-\eta_{2}\right]+E_{2}\left(T_{3}-T_{2}\right) \\
& +C_{3}^{T}\left(j \omega I-A_{3}\right)^{-1}\left[\left(T_{4}-T_{3}\right) B_{3}-\eta_{3}\right]+E_{3}\left(T_{4}-T_{3}\right)
\end{aligned}
$$




$$
\left.+C_{4}^{T}\left(j \omega I-A_{4}\right)^{-1}\left[\left(T_{5}-T_{4}\right) B_{4}-\eta_{4}\right]+E_{4}\left(T_{5}-T_{4}\right) \hat{u}\right)
$$

Expression ( 4 - 75 ) includes the transfer functions of both control to output and input to output. Note that as discussed in the second section of this chapter, the input signal perturbations $\hat{r}_{p}$ and $\hat{u}$ appear in $H_{0}(j \omega)$. In the following steps when deriving the control to output and input to output relationships, we will individually set $\hat{r}_{p}$ and $\hat{u}$ to unity to meet the requirement of $u(t)=e^{j a x}$. The response for control to output is given when $\hat{r}_{p}=1$ and $\hat{u}=0$

$$
\begin{aligned}
H_{\infty}(j \omega) & =\frac{\mu_{4}}{T,}\left\{\left[\alpha_{1}+\left(\alpha_{2} \Gamma_{1}-\zeta_{1} h_{1}^{T} \mu_{1}\right) \beta_{1}+\left(\alpha_{3} \Gamma_{2}-\zeta_{2} h_{2}^{T} \mu_{2}\right) \beta_{2} \Gamma_{1} \beta_{1}\right.\right. \\
& \left.+\left(\alpha_{4} \Gamma_{3}-\zeta_{3} h_{3}^{T} \mu_{3}\right) \beta_{3} \Gamma_{2} \beta_{2} \Gamma_{1} \beta_{1}-\zeta_{4} h_{4}^{T} \mu_{4} \beta_{4} \Gamma_{3} \beta_{3} \Gamma_{2} \beta_{2} \Gamma_{1} \beta_{1}\right] \\
& \left.\cdot\left(I-\Gamma_{4} \beta_{4} \Gamma_{3} \beta_{3} \Gamma_{2} \beta_{2} \Gamma_{1} \beta_{1}\right)^{-1} \xi_{4}+\zeta_{4}\right\}
\end{aligned}
$$

The response of input to output is given when $\hat{u}=1$ and $\hat{r_{p}}=0$

$$
\begin{aligned}
H_{b}(j \omega) & =\frac{1}{T}\left\{\left(\left[\alpha_{1}+\left(\alpha_{2} \Gamma_{1}-\zeta_{1} h_{1}^{T} \mu_{1}\right) \beta_{1}+\left(\alpha_{3} \Gamma_{2}-\zeta_{2} h_{2}^{T} \mu_{2}\right) \beta_{2} \Gamma_{1} \beta_{1}\right.\right.\right. \\
& \left.+\left(\alpha_{4} \Gamma_{3}-\zeta_{3} h_{3}^{T} \mu_{3}\right) \beta_{3} \Gamma_{2} \beta_{2} \Gamma_{1} \beta_{1}-\zeta_{4} h_{4}^{T} \mu_{4} \beta_{4} \Gamma_{3} \beta_{3} \Gamma_{2} \beta_{2} \Gamma_{1} \beta_{1}\right] \\
& \left.\cdot\left(I-\Gamma_{4} \beta_{4} \Gamma_{3} \beta_{3} \Gamma_{2} \beta_{2} \Gamma_{1} \beta_{1}\right)^{-1} \Gamma_{4}-\zeta_{4} h_{4}^{T} \mu_{4}\right) \\
& \cdot\left(\beta_{4} \Gamma_{3} \beta_{3} \Gamma_{2} \beta_{2} \Gamma_{1} \eta_{1}+\beta_{4} \Gamma_{3} \beta_{3} \Gamma_{2} \eta_{2}+\beta_{4} \Gamma_{3} \eta_{3}+\eta_{4}\right) \\
& +\left(\alpha_{2} \Gamma_{1}-\zeta_{1} h_{1}^{T} \mu_{1}\right) \eta_{1}+\left(\alpha_{3} \Gamma_{2}-\zeta_{2} h_{2}^{T} \mu_{2}\right)\left(\beta_{2} \Gamma_{1} \eta_{1}+\eta_{2}\right) \\
& +\left(\alpha_{4} \Gamma_{3}-\zeta_{3} h_{3}^{T} \mu_{3}\right)\left(\beta_{3} \Gamma_{2} \beta_{2} \Gamma_{1} \eta_{1}+\beta_{3} \Gamma_{2} \eta_{2}+\eta_{3}\right) \\
& +C_{1}^{T}\left(j \omega I-A_{1}\right)^{-1}\left[\left(T_{2}-T_{1}\right) B_{1}-\eta_{1}\right]+E_{1}\left(T_{2}-T_{1}\right) \\
& +C_{2}^{T}\left(j \omega I-A_{2}\right)^{-1}\left[\left(T_{3}-T_{2}\right) B_{2}-\eta_{2}\right]+E_{2}\left(T_{3}-T_{2}\right) \\
& +C_{3}^{T}\left(j \omega I-A_{3}\right)^{-1}\left[\left(T_{4}-T_{3}\right) B_{3}-\eta_{3}\right]+E_{3}\left(T_{4}-T_{3}\right) \\
& \left.+C_{4}^{T}\left(j \omega I-A_{4}\right)^{-1}\left[\left(T_{5}-T_{4}\right) B_{4}-\eta_{4}\right]+E_{4}\left(T_{5}-T_{4}\right)\right\}
\end{aligned}
$$


( 4 - 76) and ( 4 - 77) are transfer functions for a specific case, in which the number $N_{s}$ of topologies is 4 . We can, through observation, write the expressions for any $N_{s}$. This makes it possible to write a program which can be used to automate the derivation for the general case. The analysis procedure has been automated in a power electronic circuit simulator, named PECS [3]. Now instead of facing a tedious job of deriving the transfer functions for a specific converter, one can easily use the available CAD application to perform the dynamic analysis. The flow diagrams of the program are shown in Figure 7 and Figure 8.

\section{THE VERIFICATION OF THE RESULTS}

The theory of the time varying transfer function, as well as a variety of other related material, are provided by the previous research [ 1 ] and [ 2 ]. It is based on these research that the extended research and application presented in this thesis could be achieved.

The previous research [ 1 ], using the time varying transfer function in the frequency analysis of switched networks performed by Tymerski, includes derivations using the exact technique to find the control to output transfer function for a variety of DC to DC converters. Among the examples included are voltage mode and current programmed PWM converters operating in both CCM and DCM mode. One circuit diagram of these examples, a PWM boost converter operating in DCM, is given in Figure 9. The control to output frequency response curves for both magnitude and phase based on the result of hand-derivation are shown in Figure 10, where they are drawn as the dashed lines. To verify the accuracy of the exact technique this converter circuit was implemented. Its control to output frequency response was measured. The curves obtained by experimental measurement is also depicted in same plot with that of derivedby hand, where the former is drawn in the solid lines. The fact of both lines being 


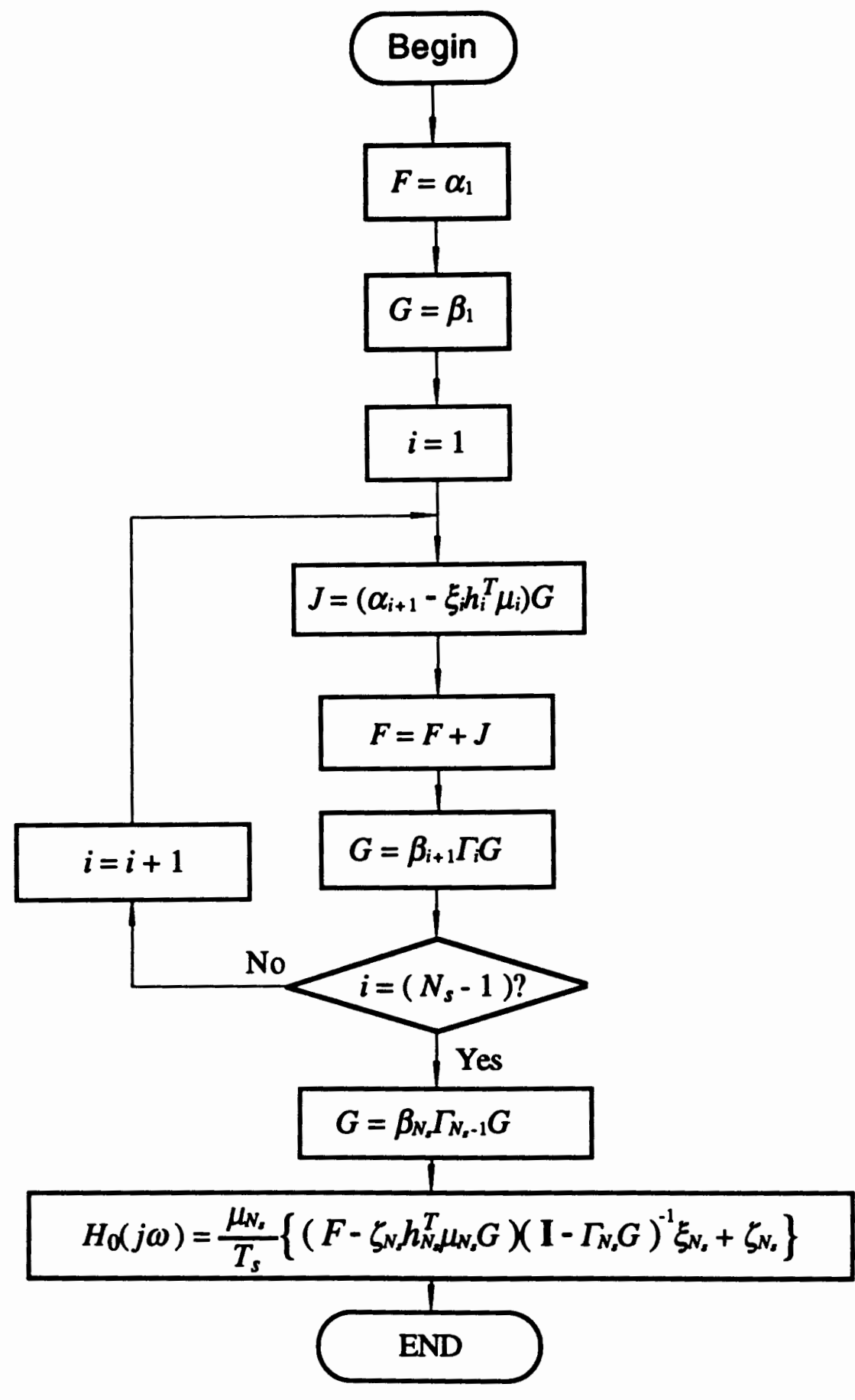

Eigure 7. Program flow diagram of control to output transfer function 


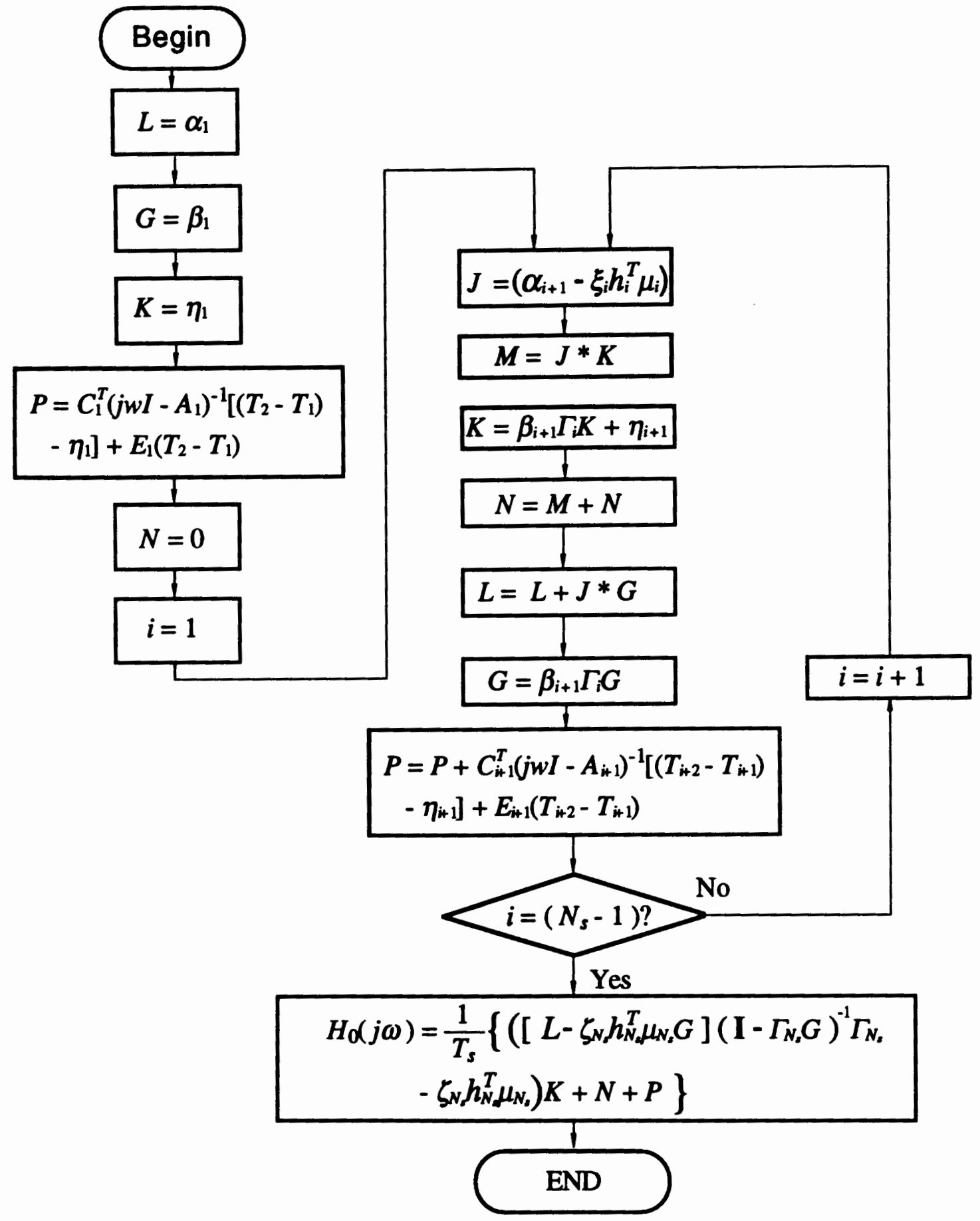

Figure 8. Program flow diagram of input to output transfer function 
being very close shows that the exact technique does feature an unparalleled accuracy.

The next example involves a current programmed PWM boost converter operating in continuous conduction mode (CCM). The circuit diagram is given by Figure 13. The procedure of deriving the control to output frequency response by hand, implementing the converter circuit and performing the measurement are exactly the same as those carried out in the first example. The two sets of frequency response curves were compared in Figure 14. The good matches strengthen the conclusion of the last example.

The price paid for achieving this high accuracy is the sheer complexity of the derivation. The procedures of the derivation diverse greatly from one converter to another. Lack of generality prevents a wide application of this technique. The remedy of this problem, which is the major part of object studied in this thesis, is an algorithm implemented in a computer. Given any specific switched network, i.e., once the state space representation of each possible topologies are found, an output in form of Bode plot will be readily available. Further more another important frequency response describing the input to output relationship is also obtained, and the derivation of which is also automated by a computer program. Although in this thesis the fluctuating power source is assumed as this disturbance input signal, the output response caused by any disturbance in the switched network can also be found by executing this program, as long as the state space representation for this case can be found.

The validity of general exact small-signal frequency analysis technique was verified by applying its programmed version to those two examples carried out in [ 1 ], because the frequency responses for those specific networks derived by hand had been proved a sound representation of the system dynamics. The output frequency curves of the PWM boost converter operating in DCM produced by this program is given by Figure 11, which entirely overlap those derived and verified by Tymerski in [ 1 ]. The same method was also applied to find the input to output transfer function. The output 
response curves of the program for this transfer function are shown in Figure 12. For the second example, which is the PWM current programmed boost converter, the frequency response of control to output produced by the computer is depicted in Figure 15. The conclusion of the comparison made between this one and the previous results, together with the one of last example, confirms that these programs provide an exact representation of the hand-derivation, and furthermore, a more useful analysis measure. Also illustrated in Figure 16 is the input to output frequency response of the current programmed boost converter. 


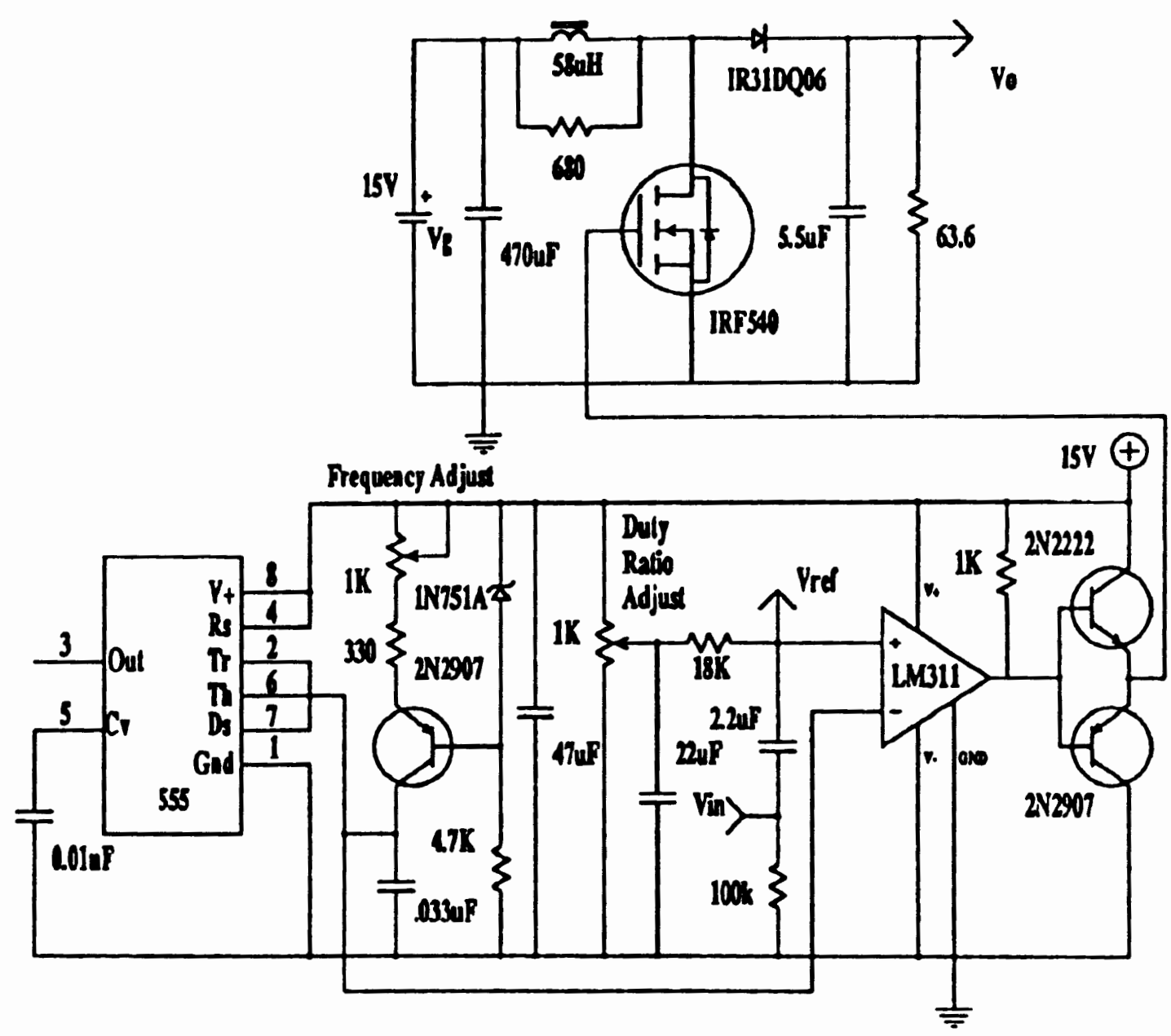

Figure 9. The circuit of a PWM boost converter operating in DCM. 

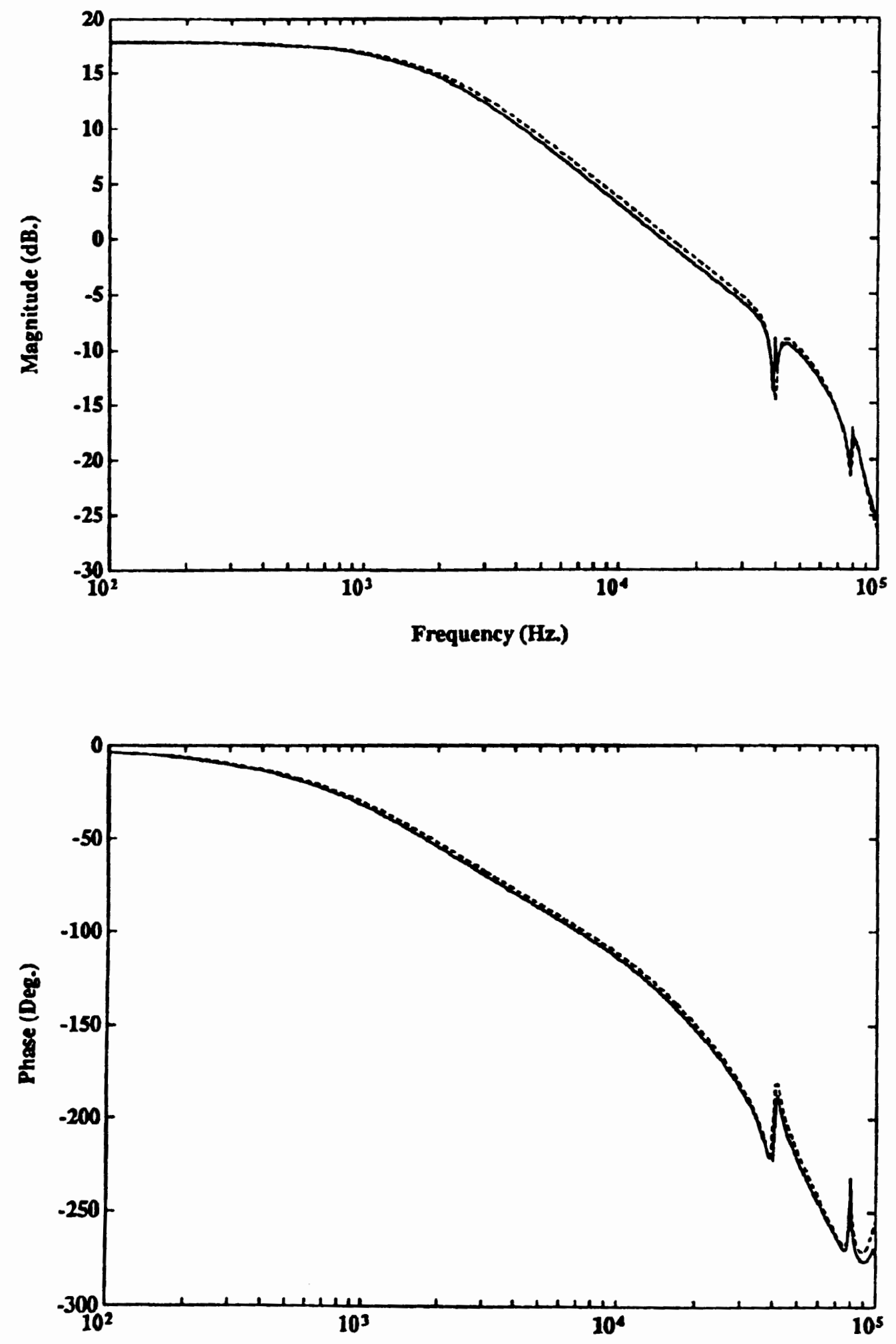

Eigure 10. The control to output frequency response of the PWM boost converter operating in DCMverified by measurement. 

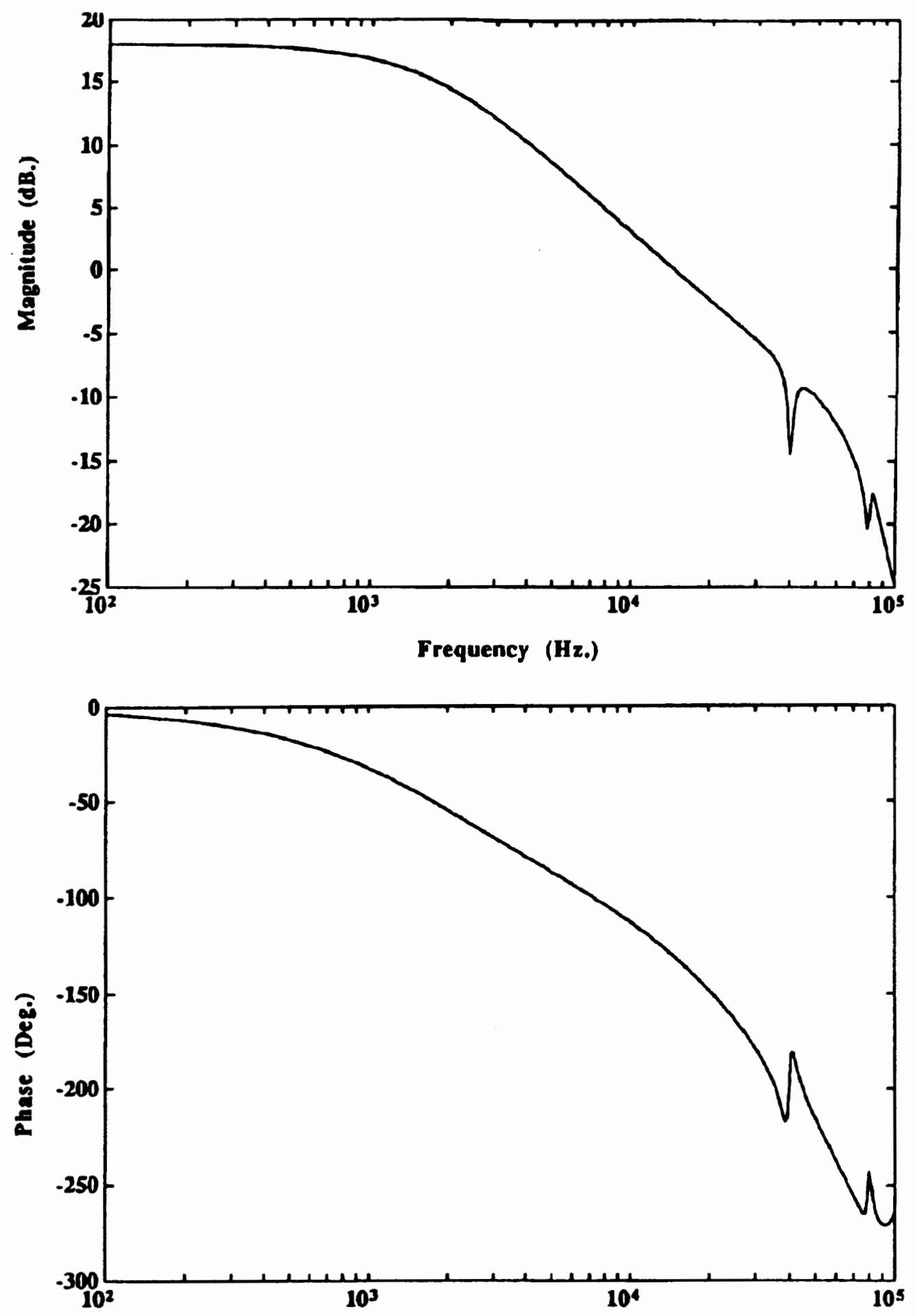

Figure 11. The control to output frequency response of the PWM boost converter operating in DCM 

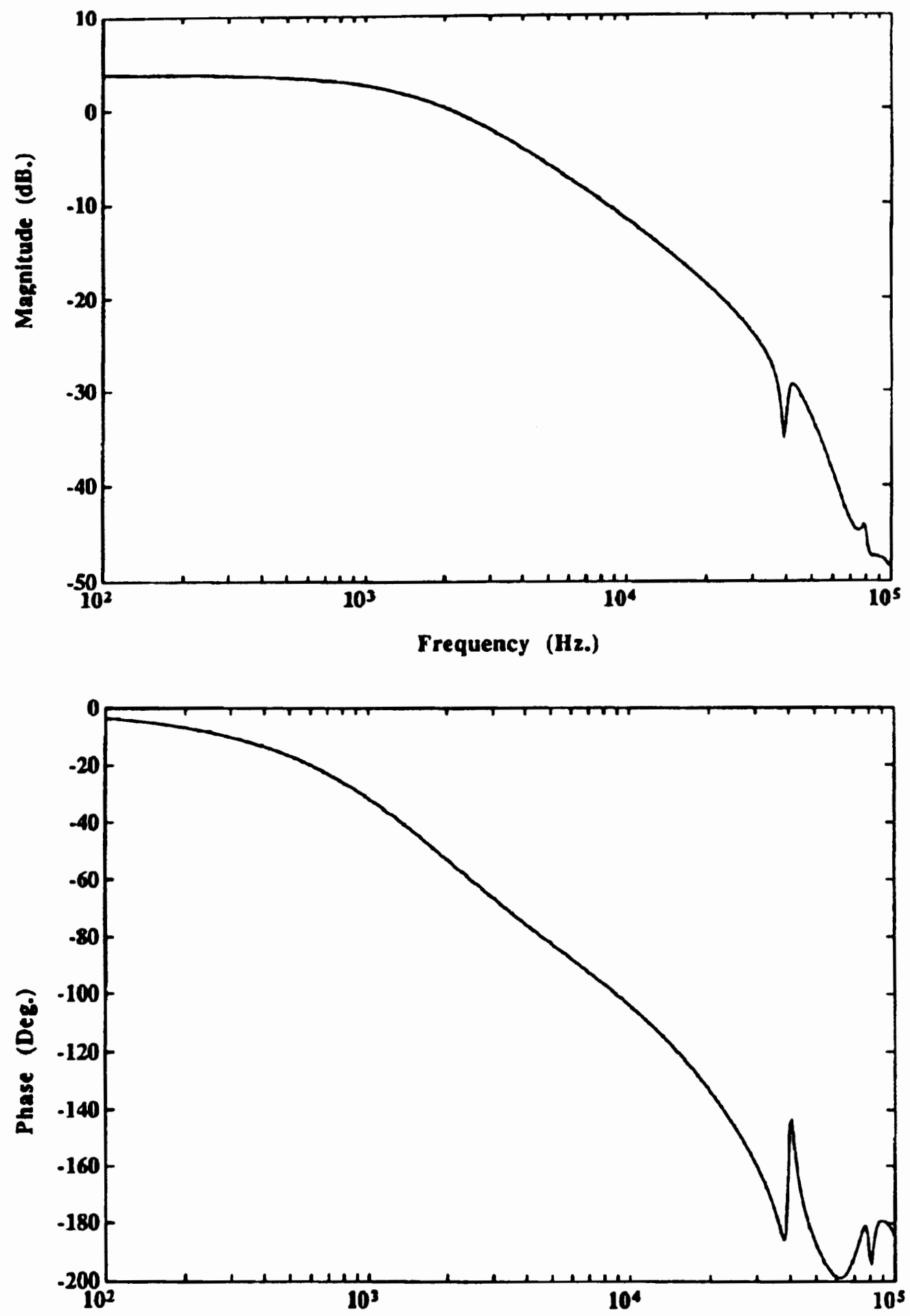

Figure 12. The input to output frequency response of the PWM boost converter operating in DCM. 


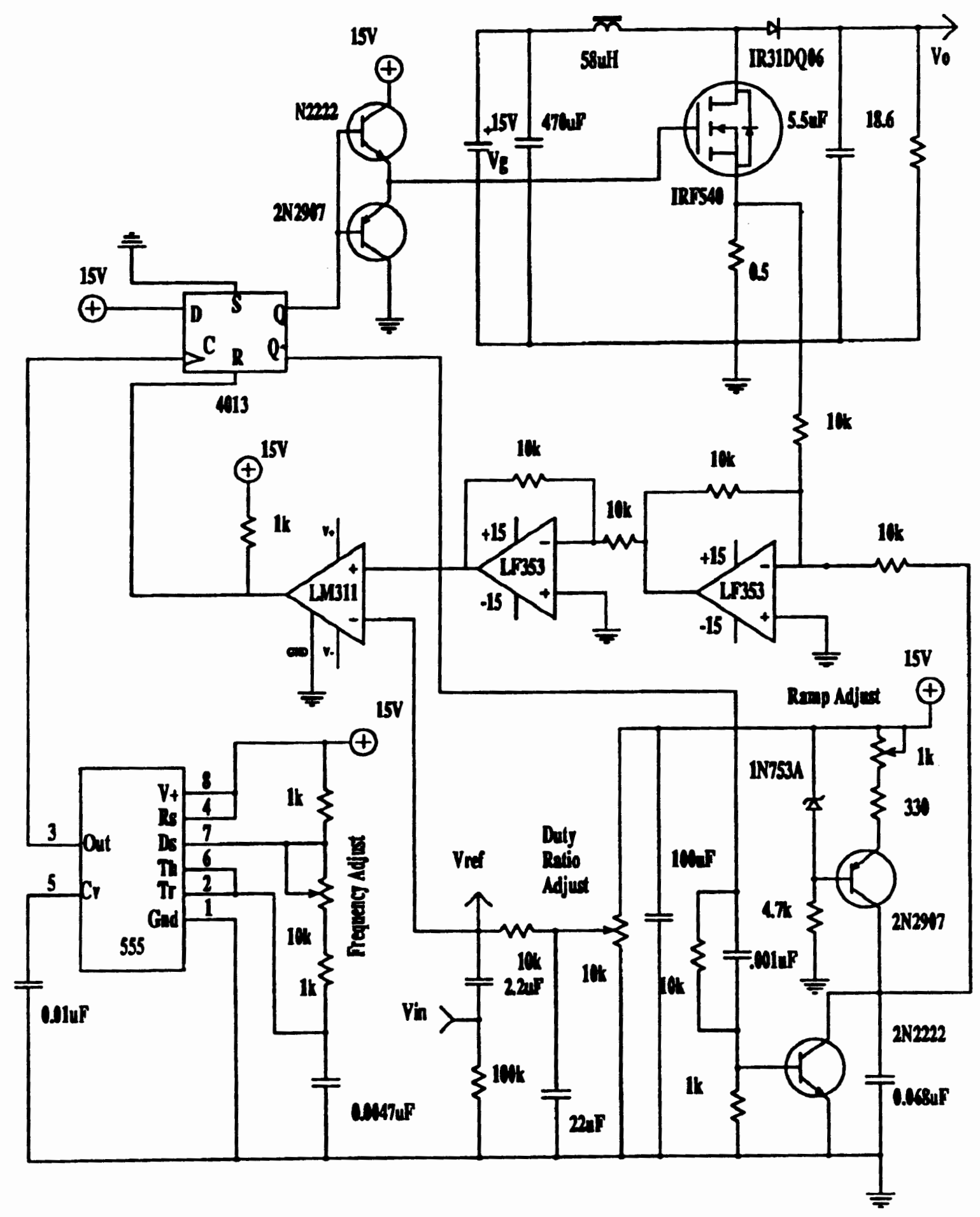

Figure 13. The circuit of a current programmed PWM boost converter operating in CCM. 

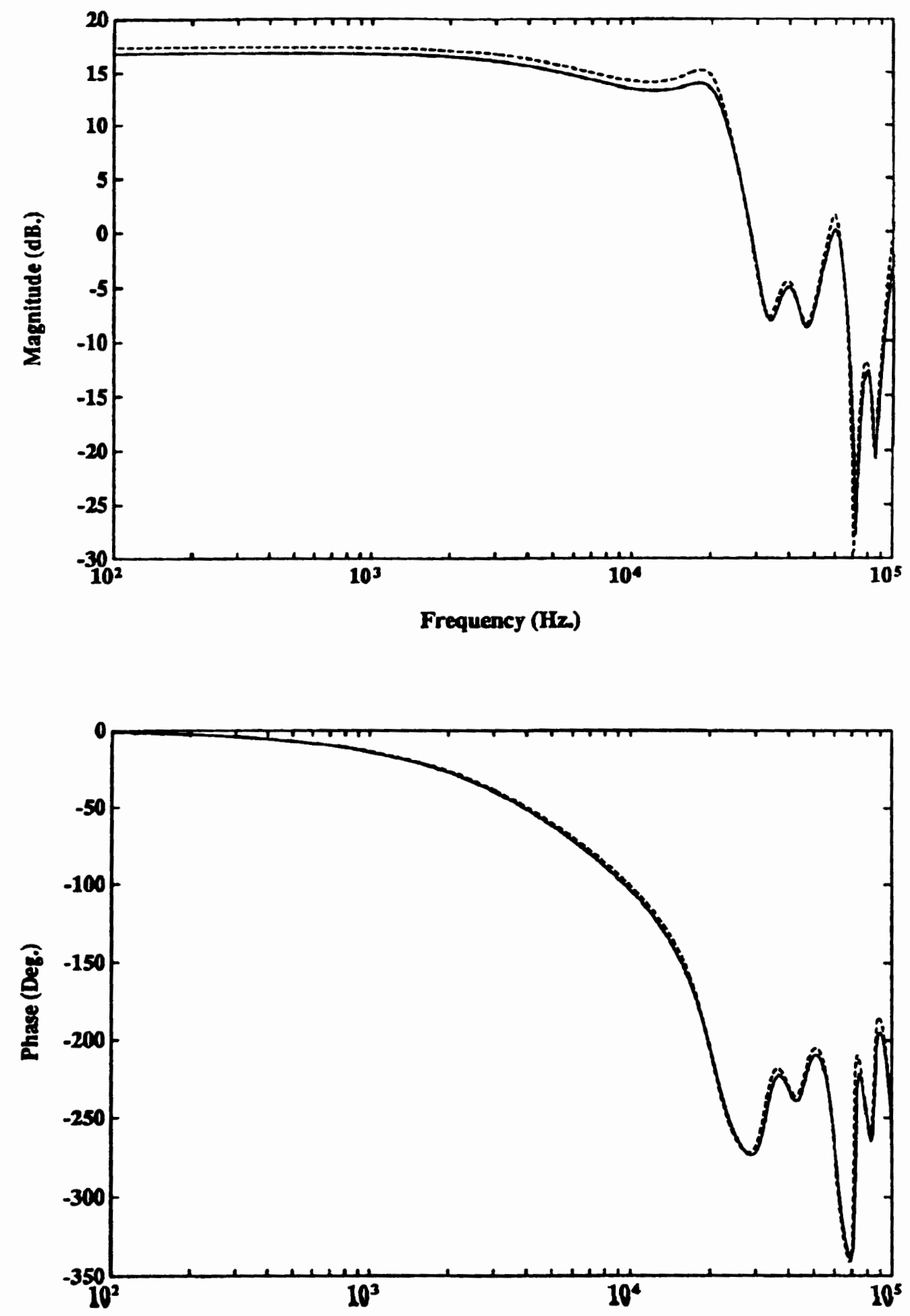

Eigure 14. The control to output frequency response of the current programmed PWM boost converter operating in CCM verified by measurement. 

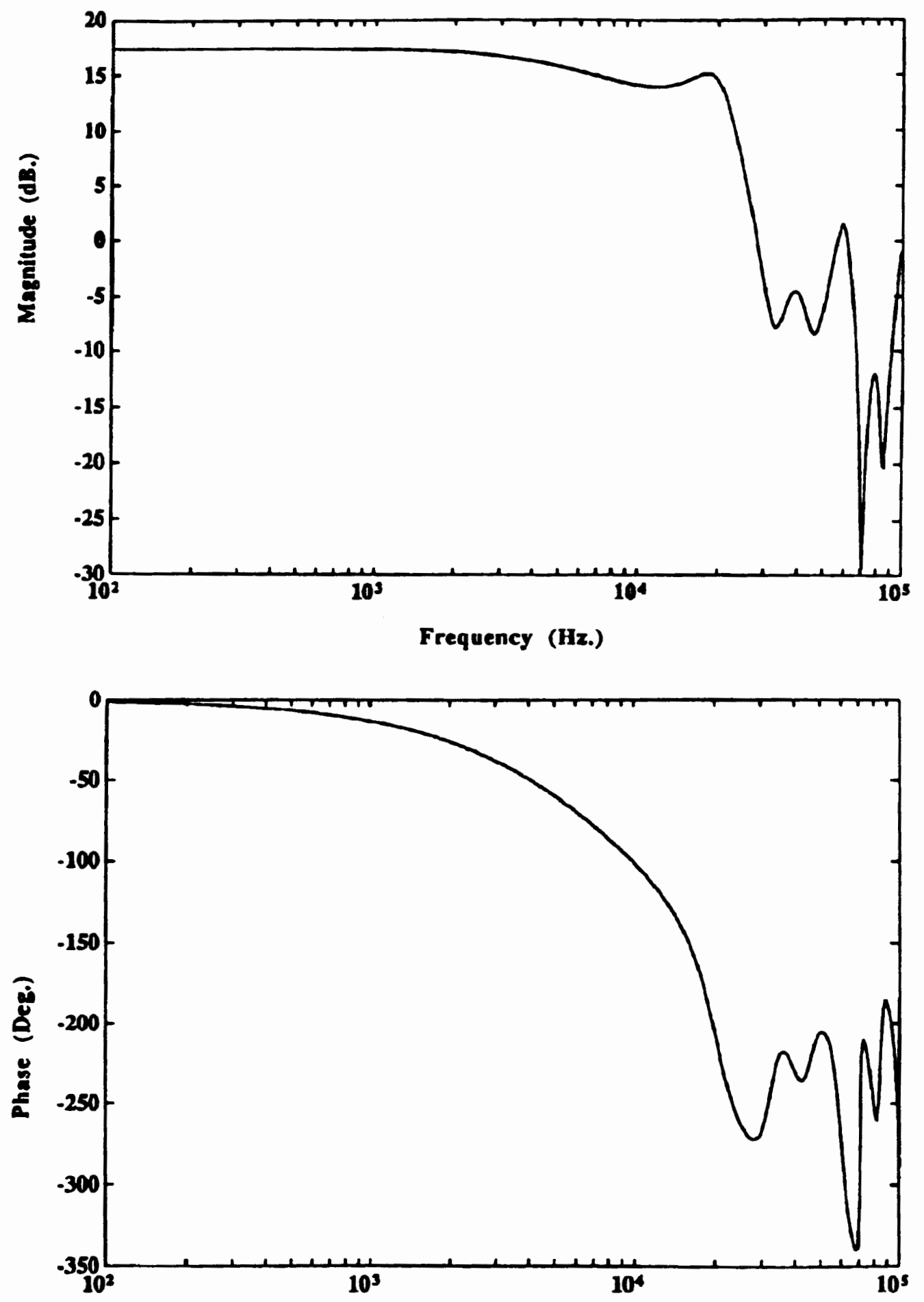

Eigure 15. The control to output frequency response of the current programmed PWM boost converter operating in CCM. 

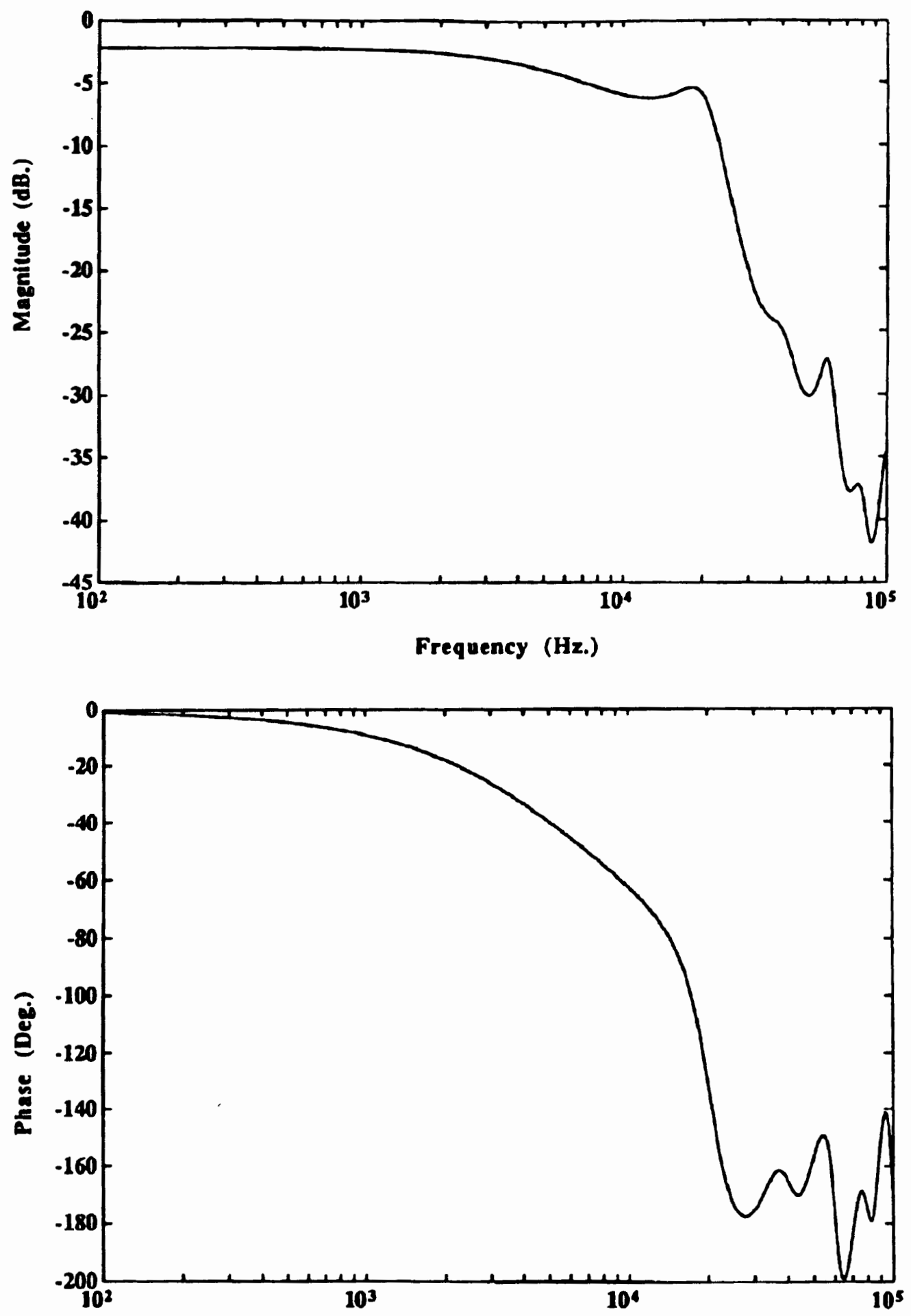

Eigure 16. The input to output frequency response of the current programmed PWM boost converter operating in CCM. 


\section{CHAPTER V}

\section{CONCLUSION}

Two frequency response techniques for analyzing switching converters are investigated. A linearized small signal model is obtained. The steady state operating points are found, which is fundamental for small signal modeling.

The sampled-data method features a discrete time mathematical description. The analytical description of converter waveforms is approached by formulating a difference equation which describes the propagation from switching cycle to cycle of a point on a converter waveform. The accuracy in high frequency range is restricted due to the fact that since the signal is sampled at a fixed time interval it is unable to take sufficient information from a signal waveform the frequency of which is higher than half the sampling frequency.

A frequency analysis of time-interval-modulated piecewice linear switched networks is introduced. The time varying transfer function is defined and, through a Fourier analysis, related to the frequency response of switched network. Over an assumed system varying time interval a lengthy derivation is performed. The state is evaluated at the end of each subinterval. The values obtained in one system varying period are summed up and divided by the period length to find the average value. Every detail happened is taken account. The benefit for paying this price is that the whole procedure will end up with an accurate frequency response in any arbitrary frequency value. To overcome the difficulty of sophisticated derivation for a specific converter, an algorithm for a CAD application is developed to automate the procedure. The output of this procedure includes steady states and switching over instants. The dynamic behavior 
of the network is performed by applying the exact small-signal frequency analysis technique. The accurate and readily available frequency response provides us with a powerful tool in analysis and design of various kinds of converters. The application of this technique allows an engineer to investigate both steady state and frequency response of his design without costly hardware prototyping. 


\section{REFERENCES}

[ 1 ] Richard Tymerski, "Frequency analysis of time-interval-modulated switched networks," IEEE Transactions on Power Electronics, Vol. 6, No. 2. April 1991.

[2 ] Richard Tymerski, "Application of the time varying transfer function for exact small-signal analysis," IEEE Power Electronics Specialists Conference (PESC) ‘91, June, 1991.

[ 3 ] Richard Tymerski, "A Fast Time Domain Simulator for Power Electronic Systems," IEEE Applied Power Electronics Conference (APEC) ‘93, San Diego, CA, March, 1993. 